\title{
Trading Volume and Information Distribution in a Market-clearing Framework *
}

\author{
Dominique Dupont \\ Division of Monetary Affairs \\ Board of Governors of the Federal Reserve System
}

August 1997

${ }^{*}$ Mail Stop 69, Federal Reserve Board, Washington, DC 20551; phone: (202) 452-6483; fax: (202) 4522301; e-mail: m1dyd99@frb.gov. The views expressed herein are the author's and do not necessarily reflect those of the Board of Governors or the Federal Reserve System. 


\begin{abstract}
This paper investigates the relations between aggregate trading volume and information on financial markets from a theoretical standpoint. Through numerical examples, it relates some statistics describing equilibrium price and volume-such as the variance of the price and its correlation with the true asset value, the volume mean, variance, skewness, and kurtosis-to the distribution of information across traders. The analysis is carried out in a static noisy rational expectations framework, with multiple informed traders, where both the precision and the correlation of the signals observed by the traders can be modified.

Numerical examples show that the variance of the market-clearing price, and the mean and variance of the volume are increasing in the precision of the informed trader's signals and-to a lesser extent-in the liquidity shock variance. The price informativeness is increasing in the precision of the informed traders' signals and decreasing in the liquidity shock variance. Skewness and kurtosis in the trading volume distribution are not always associated with a high precision of the informed trader' signals; the relation depends on the correlation across the informed traders' signals.
\end{abstract}




\section{Introduction}

Trading volume has received a lot of attention from practitioners and academics alike. Technical analysts use trading volume as an indicator that "new and compelling information is flowing into the market" (Saitta (1996)). Regulators have recently contemplated using trading volume as a tool to detect market manipulations. This seems to warrant further theoretical efforts to investigate the relations between volume and information.

The paper's objective is to relate the patterns of aggregate volume to the underlying distribution of information across traders from a theoretical perspective. It sets out to relate some important descriptive statistics of volume, such as its mean, variance, skewness, and kurtosis-together with some price statistics-to the distribution of information among traders, i.e., the precision of their information and the degree of agreement in their valuations of the asset. The analysis is carried out in a noisy rational expectations framework with a competitive securities market and multiple informed traders. When closed-form solutions for the equilibrium price are not available, a simple numerical method is introduced to compute noisy rational expectations equilibria with a flexible parameter structure.

The present approach follows the seminal work of Diamond and Verrechia (1981) and the more recent papers by, among others, Grundy and McNichols (1989), and Brown and Jennings (1989). The present model features a competitive securities market with multiple informed traders and a liquidity trader. One contribution of the paper is that the signals observed by the informed traders can be both correlated and have different precisions. This makes it easier to study the the heterogeneity of beliefs, a key element in understanding the behavior of trading volume. In Diamond and Verrechia (1981), Brown and Jennings (1989), and He and Wang (1995), the signals observed by the informed traders are independently and identically distributed, when conditioned on the true value of the asset. ${ }^{1}$ In Grundy and McNichols (1989), the signals are correlated across traders but have the same precision. In a different framework, Blume, Easley and O'Hara (1994) use signals with varying precisions. As pointed by Jain (1988), the response of trading volume to economic news may indicate the degree to which market participants disagree about the effect of the announcement. With identically distributed signals, the heterogeneity of beliefs is ex-post, i.e., the realizations of the privately observed signals may be different across agents even though the signals are all independently drawn from the same distribution. In an ex-ante approach, the focus is shifted from the difference in the realization of the signals to the difference in their distribution. The heterogeneity of beliefs depends on the degree of disagreement among traders about the true asset value and on the difference in the precision of the information they possessed.

\footnotetext{
${ }^{1} \mathrm{He}$ and Wang note that "extensions to more general correlation structure, except for the correlation between the signals of different investors, are quite straightforward," (p. 925) [emphasis added].
} 
Analytically, the degree to which the traders agree about the value of the asset is determined by the correlation between their private signals, the quality of the traders' information is determined by the correlation of their signals with the asset value. Allowing signals to be correlated and have different precisions makes it easier to investigate economically relevant problems, like the effect of a public announcement versus the effect of private information. Public information would translate in the model as a high level for the signals' correlation. More private information could be modeled by increasing the precision of one trader's information without changing the precision of the other traders' signals. When the informed traders' signals have different precision, the model cannot be solved analytically and numerical solutions are used.

Trade size and private information have often been related in the literature. For example, Easley and O'Hara (1987), in a market-maker setup, point out that "trade size introduces an adverse selection problem into security trading, because given that they wish to trade, informed traders prefer to trade large amounts at any given price" (p.69). In the present model, the quality of traders' information can be related to the fatness of tails, as one may conjecture that the probability of large trades would increase with the quality of the traders' information. Looking at the volume kurtosis seems a good starting point. As far as large trades would make the volume more asymmetric, the same case could be made for the skewness. Interestingly enough, the results show that the effect of an increase in the information quality of one or more traders on the volume skewness and kurtosis is very sensitive to the the degree of agreement among the traders as measured by the coefficient of correlation between their signals. Depending on the value of this parameter, skewness and kurtosis could be low while the traders are well informed, and high when they are not. Foster and Viswanathan (1996), in a model with multiple, differentially informed traders, also found that "the trading outcomes depend critically on the initial correlation of the informed traders' private signals" (p. 1439). However, the correlation of the informed traders' signals has little effect on the mean and variance of the volume. The paper borrows techniques classically used by the noisy rational expectations literature, such as negative exponential utility, normally distributed random variables, and some noise trading. However, in the paper, the noise does not stem from an exogenous random asset supply, as is often the case in the literature, but from a price-sensitive liquidity demand.

The first section of the paper introduces the model. The second section presents numerical examples based on different assumptions about the distribution of information across traders.

\section{Model}

The paper uses a static rational expectations framework with one liquidity trader and $n$ informed traders, each observing a signal $G_{i}, i=1, \ldots, n$, correlated with the true value of the asset. For each index $i$, the random variable $G_{i}$ has mean zero and variance one, the vector $\left(G_{1}, \ldots, G_{n}, x\right)$ is 
normally distributed. Each trader has a CARA utility function $u_{i}\left(\pi_{i}\right)$ with risk aversion coefficient $\lambda_{i} .{ }^{2}$ Agent $i$ 's profit, $\pi_{i}$, is the random variable $y_{i}(x-p)$, where $y_{i}$ is the demand of trader $i, x$ the true value of the asset, and $p$ the market price. The $(n+1)^{t h}$ trader is a noise trader, whose demand is $y_{n+1}=-b(p-\varepsilon)$, where $b>0$, and $\varepsilon$ is uncorrelated with the true value of the asset. In the simulations, unless specified otherwise, $\operatorname{var}(x)$ equals $\operatorname{var}(\varepsilon)$, which would be the variance of the market-clearing price with no informed traders.

Informed traders submit demand schedules as functions of the market-clearing price. Each informed trader chooses $y_{i}$ to maximize $E\left[u\left(\pi_{i}\right) \mid G_{i}, p\right]$. The framework described above must be seen as a 'reduced form' of a structural model, which would introduce another period where agents consume but do not trade, and a riskless asset that traders could purchase or sell to finance to desired holdings of risky securities, as in Diamond and Verrechia (1981).

Let $v_{i}=E\left[x \mid G_{i}, p\right]$ and $\sigma_{i}^{2}=\operatorname{var}\left[x \mid G_{i}, p\right]$. As $\left(p, G_{1}, \ldots, G_{n}\right)$ is normally distributed, informed trader $i$ 's demand is $y_{i}(p)=\alpha_{i}\left(v_{i}-p\right)$, with $\alpha_{i}=\frac{1}{\lambda_{i} v a r\left[x \mid G_{i}, p\right]}$. The agent takes into account that $p$ is the market-clearing price, i.e., $p=\Sigma_{1}^{n} \theta_{i} v_{i}+\theta_{n+1} \varepsilon$, with $\alpha=\Sigma_{1}^{n} \alpha_{i}, \theta_{i}=\frac{\alpha_{i}}{b+\alpha}$, for $i=1, \ldots, n$, and $\theta_{n+1}=\frac{b}{b+\alpha}$ (in the applications, $b=1$ ). Note that $\Sigma_{i=1}^{n+1} \theta_{i}=1, \theta_{i}$ is the weight of the $i^{t h}$ trader in the determination of the market-clearing price. As a result, trader i's equilibrium valuation is $v_{i}$ such that

$$
v_{i}=E\left[x \mid G_{i}, \Sigma_{j=1}^{n} \theta_{j} v_{j}+\theta_{n+1} \varepsilon\right]
$$

Equation (1) is an example of 'infinite regress'. For any two agents $i$ and $j$, agent $i$ 's valuation depends on agent $j$ 's valuation, in turn, agent $j$ 's valuation depends on agent $i$ 's valuation, and so forth. Besides, in (1), traders have to take into account the 'noise' injected in the trading by $\varepsilon$. Equilibrium valuations can be thought of as solutions to $n$ simultaneous equations similar to (1), with $i=1, \ldots, n$. Solutions to this system of equations may fail to exist. The limit to infinite regress is common knowledge, which, in the absence of noise, can result in an equilibrium where no trade takes place, as in Milgrom and Stockey (1982). As proposition (1) shows, if there is a normally distributed solution $v^{*}$ to $(1)$, then the equilibrium price is linear in the traders signals and the liquidity noise.

Proposition 1 If there is a vector $v^{*}$ satisfying (1) and $v^{*}, G, x$ and $\varepsilon$ are jointly normally distributed, the corresponding equilibrium price $p^{*}$ is linear in the informed traders' signals and the noise trader's liquidity shock, that is,

$$
p^{*}=a_{1}^{*} G_{1}+a_{2}^{*} G_{2}+a_{3}^{*} \varepsilon .
$$

Proof of proposition 1: Suppose there is a normally distributed fixed point $z^{*}=\left(v^{*}, G, \varepsilon, x\right)$. Recall

\footnotetext{
${ }^{2}$ In the numerical examples, the risk aversion coefficient is assumed to be equal to one.
} 
that $p^{*}=\sum_{i=1}^{n} \theta_{i}^{*} v_{i}^{*}+\theta_{n+1}^{*} \varepsilon$. Consequently, $z_{i}^{*}=\left(G_{i}, p^{*}\right)$ is a normally distributed vector, and $v_{i}^{*}=E\left[x \mid G_{i}, p^{*}\right]$ is linear in $G_{i}$ and $p^{*}$. Writing $v_{i}^{*}=c_{i} G_{i}+d_{i} p^{*}$, we get that $p^{*}$ verifies the following equation

$$
\left(1-\Sigma_{i=1}^{n} \theta_{i} d_{i}\right) p^{*}=\Sigma_{i=1}^{n} \theta_{i}^{*} c_{i} G_{i}+\theta_{n+1}^{*} \varepsilon
$$

Suppose that $\sum_{i=1}^{n} \theta_{i}^{*} d_{i}=1$, then $\varepsilon$ and the $G_{i}$ would be correlated, which contradicts the assumption that the liquidity shock is uncorrelated with the signals. Hence, $\sum_{i=1}^{n} \theta_{i}^{*} d_{i} \neq 1$, and $p^{*}$ can be written as a linear combination of the state variables. When the precision of the signal is equal across traders, the coefficient $a_{1}^{*}, a_{2}^{*}, a_{3}^{*}$ have a closed form solution. Otherwise, one has to use numerical techniques.

Often in the literature (for example, Diamond and Verrechia (1981), Wang (1994)) each trader's private signal is assumed to be the sum of the true asset value and a 'measurement error' term, assumed to identically and independently distributed across traders (in that sense, the signals are conditionally identically and independently distributed, when conditioned on the true asset value). In the present formulation, this boils down to having $\rho_{1}=\rho_{2}=\rho^{2} .{ }^{3}$ The 'measurement error' approach doe not allow changing the precision without affecting the correlation of the signals.

\subsection{Equilibrium with two informed traders with equally precise signals}

\subsubsection{Rational expectation equilibrium}

Assume that the informed trader' signals $G_{1}$ and $G_{2}$ are correlated and have the same covariance with the true value of the traded asset, i.e., they have the same precision. Let $\gamma=E\left[G_{1} G_{2}\right]$ and $\rho=E\left[x G_{1}\right]=E\left[x G_{1}\right]$. Since the vector state vectors $\left(G_{1}, G_{2}, x, \varepsilon\right)$ and $\left(G_{2}, G_{1}, x, \varepsilon\right)$ have the same distribution, $G_{1}$ and $G_{2}$ must have the same coefficient in equation (2). We can write

$$
p=a\left(G_{1}+G_{2}\right)+b \varepsilon
$$

The equilibrium price is also equal to an weighted average of the trader's valuation of the asset and the liquidity noise.

$$
p=\theta\left(v_{1}+v_{2}\right)+(1-2 \theta) \varepsilon
$$

where $\theta=\frac{\alpha_{1}}{1+\alpha_{1}+\alpha_{2}}$, and $\alpha_{1}=\alpha_{2}=\frac{1}{\operatorname{var}\left(x \mid G_{1}, p\right)}=\frac{1}{\operatorname{var}\left(x \mid G_{2}, p\right)}$, which implies that $\theta=\frac{1}{2+\operatorname{var}\left(x \mid G_{1}, p\right)}=$ $\frac{1}{3-E\left[v_{1}^{2}\right]}$. The informed traders' valuations $v_{1}, v_{2}$ and the weight $\theta$ depend on the coefficients $a$ and $b$ in equation (4). The key is to find values for $a$ and $b$ so that equations (4) and (5) hold

\footnotetext{
${ }^{3}$ To see this, let the private signal of trader $i$ be $g_{i}=x+\eta_{i}$. The $\eta_{i}$ are i.i.d with variance $\sigma_{\eta}^{2}$ and are uncorrelated with $x$. All the variables are jointly normally distributed. As $E\left[x g_{i}\right]=E\left[g_{i} g_{j}\right]=\sigma_{x}^{2}$, for $i \neq j$, and $E\left[g_{i}^{2}\right]=\sigma_{x}^{2}+\sigma_{\eta}^{2}$ $\operatorname{corr}\left(g_{i}, x\right)=\frac{\sigma_{x}}{\sqrt{\sigma_{x}^{2}+\sigma_{\eta}^{2}}}, \operatorname{corr}\left(g_{i}, g_{j}\right)=\frac{\sigma_{x}^{2}}{\sigma_{x}^{2}+\sigma_{\eta}^{2}}$.
} 
simultaneously. Trader 1's equilibrium valuation is $v_{1}$ such that,

$$
\begin{aligned}
v_{1} & =E\left[x \mid G_{1}, p\right] \\
& =E\left[x \mid G_{1}, a\left(G_{1}+G_{2}\right)+b \varepsilon\right] \\
& =E\left[x \mid G_{1}, G_{2}+\frac{b}{a} \varepsilon\right] \\
& =E\left[x \mid G_{1}, G_{2}-\gamma G_{1}+\frac{b}{a} \varepsilon\right] \\
& =E\left[x \mid G_{1}\right]+E\left[x \mid G_{2}-\gamma G_{1}+\frac{b}{a} \varepsilon\right] \\
& =\rho G_{1}+\frac{\operatorname{cov}\left(x, G_{2}-\gamma G_{1}+\frac{b}{a} \varepsilon\right)}{\operatorname{var}\left(G_{2}-\gamma G_{1}+\frac{b}{a} \varepsilon\right)}\left(G_{2}-\gamma G_{1}+\frac{b}{a} \varepsilon\right) \\
& =\rho G_{1}+\frac{\rho(1-\gamma)}{1+\gamma^{2}+(b / a)^{2}}\left(G_{2}-\gamma G_{1}+\frac{b}{a} \varepsilon\right)
\end{aligned}
$$

The fifth line comes from the fact that $G_{2}-\gamma G_{1}$ and $\varepsilon$ are uncorrelated with $G_{1}$ and that all the variables are normally distributed. We conclude that

$$
\begin{aligned}
v_{1}+v_{2} & =\rho\left(G_{1}+G_{1}\right)+\frac{\rho(1-\gamma)^{2}}{1+\gamma^{2}+(b / a)^{2}}\left(G_{1}+G_{1}\right)+\frac{2 \rho(1-\gamma)}{1+\gamma^{2}+(b / a)^{2}} \varepsilon \\
& =\rho \frac{2(1-\gamma)+(b / a)^{2}}{1-\gamma^{2}+(b / a)^{2}}\left(G_{1}+G_{2}\right)+\frac{2 \rho(1-\gamma)}{1+\gamma^{2}+(b / a)^{2}} \varepsilon
\end{aligned}
$$

Combining equations (5) and (7), we get

$$
\begin{aligned}
\theta^{-1}\left(1-\gamma^{2}+(b / a)^{2}\right) p & =\rho\left(2\left(1-\gamma^{2}\right)+(b / a)^{2}\right)\left(G_{1}+G_{2}\right) \\
& +\left(1-\gamma^{2}-2(1-\gamma) \rho^{2}+\left(1-\gamma^{2}\right)(b / a)^{2}+2 \rho(1-\gamma)(b / a) \varepsilon\right.
\end{aligned}
$$

To compute $\theta$, note that $E\left[v_{1}^{2}\right]=\rho^{2} \frac{2(1-\gamma)+(b / a)^{2}}{1-\gamma^{2}+(b / a)^{2}}$. Hence, $b / a$, the ratio of the coefficient on $\varepsilon$ to the one on $G_{1}+G_{2}$, must be such that

$$
b / a=\frac{-2(1-\gamma) \rho^{2}-\rho^{2}(b / a)^{2}+1-\gamma^{2}+(b / a)^{2}+2 \rho(1-\gamma)(b / a)}{2 \rho(1-\gamma)+\rho(b / a)^{2}}
$$

Write $\beta=b / a$, then, the equilibrium $\beta$ must solve the following cubic equation.

$$
\varphi(\beta, \rho, \gamma)=\rho \beta^{3}-\left(1-\rho^{2}\right) \beta^{2}+(1-\gamma)\left(2 \rho^{2}-\gamma-1\right)=0
$$

For $\gamma$ and $\rho$ which yield a positive definite covariance matrix for the state vector $\left(G_{1}, G_{2}, x, \varepsilon\right)$, there is a unique real solution to the previous cubic equation. ${ }^{4}$ Call $a^{*}(\rho, \gamma)$ and $b^{*}(\rho, \gamma)$ the resulting equilibrium coefficients in the pricing equation. When $\gamma=1$, the signal of the informed traders are perfectly correlated and $a^{*}$ and $b^{*}$ collapse to their values in the one-informed-trader case, and

${ }^{4}$ The matrix $\left(\begin{array}{llll}1 & \gamma & \rho & 1 \\ \gamma & 1 & \rho & 0 \\ \gamma & \gamma & 1 & 0 \\ 0 & 0 & 0 & 1\end{array}\right)$ with $-1<\gamma<1$ and $0<\rho<1$ is the covariance matrix of $\left(G_{1}, G_{2}, x, \varepsilon\right)$ provided it is positive definite, i.e, if and only if $\gamma>2 \rho^{2}-1$. Once $\gamma$ has been chosen, $\rho$ must be between 0 and $\sqrt{\frac{\gamma+1}{2}}$. 
$p=\frac{\rho G+\left(1-\rho^{2}\right) \varepsilon}{2-\rho^{2}}$.

\subsubsection{Naive expectation equilibrium}

Researchers assume sometimes, for the sake of simplicity, that the traders are "naive", that is, their valuation of the asset is $E\left[x \mid G_{i}\right]$ instead of $E\left[x \mid G_{i}, p\right] .{ }^{5}$ The equilibrium price is then $p=\theta\left(v_{1}+v_{2}\right)+(1-2 \theta) \varepsilon$, with $\theta=\frac{1}{2+\operatorname{var}\left(x \mid G_{1}\right)}=\frac{1}{2+\operatorname{var}\left(x \mid G_{2}\right)}=\frac{1}{3-\rho^{2}}$, and $v_{i}=\rho G_{i}$, $i=1,2$. Consequently, the equilibrium price is $p^{*}=a^{*}\left(G_{1}+G_{2}\right)+b^{*} \varepsilon$, with $a^{*}=\frac{\rho}{3-\rho^{2}}$ and

$b^{*}=\frac{1-\rho^{2}}{3-\rho^{2}}$. Those are the same coefficients as the ones obtained with two rational traders and perfectly correlated signals $(\gamma=1)$. This shows that assuming that the traders are naive can lead to some serious misspecifications.

Figure (1) displays the coefficients $a$ and $b$ as a function of $\rho$ for $\gamma=0$ (the solid line) and $\gamma=1$ (the dotted line) obtained with rational traders, with $\gamma=1$ representing also the naive traders' case. Note that when $\gamma=0$, the maximum level of $\rho$ is $\frac{1}{\sqrt{2}}$, while when $\gamma=1, \rho$ can take values up to 1 . In all cases, $a=0$ when $\rho=0$ and rises with $\rho$. The coefficient on $\varepsilon$ is a decreasing function of $\rho$ in the naive case, but can be an increasing function of $\rho$ in the rational case, and is always greater in the rational case than in the naive case. Figure (2) displays rational equilibrium $a$ and $b$ as a function of $\rho$, setting $\gamma$ equal to $-.5,0, .5$, and 1 .

\subsection{Equilibrium with two informed traders when the informed traders' signals have different precision.}

This section develops an algorithm to compute a solution to the fixed point problem for the informed traders' valuations (equation (1)). We checked that, when the information precision is equal across traders, the solutions obtained numerically are identical to the closed-form solutions.

Equation (1) can be rewritten as an evolution equation for agent $i$ 's valuation (equation (11).

$$
v_{i}^{(p+1)}=E\left[x \mid G_{i}, \Sigma_{j} \theta_{j}^{(p)} v_{j}^{(p)}+\theta_{n+1}^{(p)} \varepsilon\right]
$$

where $\theta_{i}^{(p)}=\frac{\alpha_{i}^{(p)}}{b+\alpha^{(p)}}$, for $i=1, \ldots, n, \theta_{n+1}^{(p)}=\frac{b}{b+\alpha^{(p)}} . \alpha^{(p)}=\Sigma \alpha_{j}^{(p)}, \alpha_{i}^{(p)}=\frac{1}{\lambda_{i} \sigma_{i}^{(p)}}, \sigma_{i}^{(p)}$ being the conditional variance of $x$ conditioned on agent $i$ information in the $p^{\text {th }}$ run of the algorithm.

$$
\sigma_{i}^{(p)}=E\left[\left(x-v_{i}^{(p)}\right)^{2}\right]=E\left[x^{2}\right]-E\left[\left(v_{i}^{(p)}\right)^{2}\right]
$$

Let $v^{(p)}=\left(v_{i}^{(p)}\right)_{i=1, \ldots, n}^{\prime}$, and $\sigma^{(p)}=\left(\left[\sigma_{i}^{(p)}\right]^{2}\right)_{i=1, \ldots, n}^{\prime}$. Given $v^{(p)}$, equation (11) determines $v^{(p+1)}$, the $\sigma^{(p)}$ used to compute the weights are obtained from equation (12). Moreover, if $v^{(p)}$ is normally distributed, so is $v^{(p+1)}$. Let $H$ be the mapping defined by $v^{(p+1)}=H\left(v^{(p)}\right)$. If $H$ has a fixed

\footnotetext{
${ }^{5}$ This is done mostly in dynamic models when the rational expectation equilibrium would be intractable.
} 
point, called it $v^{*}$, then $v^{*}$ solves (1). To simplify computations, define a new state vector $z^{(p)}$. Let $z^{(p)}=\left(v^{(p)}, G, \varepsilon, x\right)$, and $\Sigma^{(p)}=\operatorname{var}\left(z^{(p)}\right)$. The whole state of the recursive updating of expectation is described by $\Sigma^{(p)}$.

Choose as initial value $v^{(0)}$ the normally distributed vector $\left(E\left[x \mid G_{i}\right]\right)_{i=1}^{n}$; by induction, the $v^{(p)}$ are normally distributed for all $p$ 's. Hence, one need only care about the $\Sigma^{(p)}$, which evolve according to $\Sigma^{(p+1)}=F\left(\Sigma^{(p)}\right)$. $F$ is a non-linear function due to the updating of the $\theta_{i}^{\prime} s$. I could not show the existence of a fixed point; instead, a fixed point was obtained for $F$ using numerical techniques. However, the unicity of the equilibrium is not established. In the cases studied, the fixed point matrix $\Sigma^{*}$ is nonsingular, i.e., $v^{*}$ has a non degenerate normal distribution. One can then calculate the variance-covariance matrix of the trading volume and the true value of the asset, and compute some statistics describing the trading volume. One can verify that, without informed traders, no agent trades, which is in line with the no-trade theorems. When the informed traders' signals have the same precision, the numerical solution yields the same equilibrium covariance matrix for the state vector (and the same coefficient $a$ and $b$ in (4)) as the closed-form solution.

To compute (11), note that, if $p^{(k)}$ is the market-clearing price for the $k^{\text {th }}$ run of the algorithm, $\left(G_{i}, p^{(k)}, x\right)$ is normally distributed. Hence $v_{i}^{(k+1)}=E\left[x \mid G_{i}, p^{(k)}\right]$ is the linear projection of $x$ on $\left(G_{i}, p^{(k)}\right)$. Using the state space representation, one gets $v_{i}^{(k+1)}=\Delta_{i}^{(k)} z^{(k)}$. Let $\Delta^{(k)}=\left(\Delta_{i}^{(k)}\right)_{i=1}^{n}$, $v^{(k+1)}=\Delta^{(k)} z^{(k)}$. Let $\Gamma^{(k)}=\left(\begin{array}{cc}\Delta^{(k)} & \\ 0 & I_{n+2}\end{array}\right), 0$ is a $(n+2) \times n$ vector of zeroes and $I_{n+2}$ is the $(n+2) \times(n+2)$ identity matrix. We get

$$
\begin{aligned}
z^{(p+1)} & =\Gamma^{(k)} z^{(k)} \\
\Sigma^{p+1} & =\Gamma^{(k)} \Sigma^{(k)} \Gamma^{(k)^{\prime}}
\end{aligned}
$$

Furthermore, at each iteration, $p^{(k)}$ is also linear in the state variables. The conditional variances can be recursively computed as follows.

$$
\sigma_{i}^{(k)}=\theta_{x}{ }^{\prime} \Sigma^{(k)} \theta_{x}-\theta_{v_{i}}{ }^{\prime} \Sigma^{(k)} \theta_{v_{i}}
$$

where $\theta_{x}$ and $\theta_{v_{i}}$ are selection matrices such that $x=\theta_{x} z$, and $v_{i}=\theta_{v_{i}} z$. Let $z_{0}=\left(G_{1}, G_{2}, \varepsilon, x\right)^{\prime}$ and $M_{0}=\operatorname{cov}\left(z_{0}\right)$. The elements of $z_{0}$ are the fundamental parameters defining the problem. We start the recursions with $v_{i}^{(0)}=E\left[x \mid G_{i}\right]=E\left[x G_{i}\right] G_{i}$, and $\left(\sigma_{i}^{(0)}\right)=\operatorname{var}\left(x \mid G_{i}\right)=\sigma_{x}^{2}-E\left[x G_{i}\right]^{2}$.

In the following equations, demands for the asset -as functions of the informed agents' valuations and the liquidity shock- are derived in the case of two informed traders. Formulas can be generalized 
to situations with more traders.

$$
\begin{aligned}
y_{1} & =\frac{\alpha_{1}}{b+\alpha}\left[\left(b+\alpha_{2}\right) v_{1}-\alpha_{2} v_{2}-b \varepsilon\right] \\
y_{2} & =\frac{\alpha_{2}}{b+\alpha}\left[-\alpha_{1} v_{1}+\left(b+\alpha_{1}\right) v_{2}-b \varepsilon\right] \\
y_{3} & =\frac{-b}{b+\alpha}\left[\alpha_{1} v_{1}+\alpha_{2} v_{2}-\alpha \varepsilon\right] \\
p & =\frac{-b}{b+\alpha}\left[\alpha_{1} v_{1}+\alpha_{2} v_{2}+b \varepsilon\right]
\end{aligned}
$$

Then, one can compute the variance-covariance matrix of the zero mean vector $\left(y_{1}, y_{2}, y_{3}, x, p\right)$. This matrix will be used to derive the characteristics of the trading volume. The model could be extended to study how agents with different private signals interpret public information differently. Let $u$ be a public signal observed by every trader. Agent $i$ 's equilibrium valuation $v_{i}$ becomes $E\left[x \mid G_{i}, u, p\right]$. Assuming that all random variables are normally distributed, $v_{i}=\beta_{G_{i}}^{i} G_{i}+\beta_{u}^{i} u+\beta_{p}^{i} p$. The marginal effect of the publicly released information on trader $i$ 's valuation $\beta_{u}^{i}$ depends on the correlation between $x, u, G_{i}$, and $p$, and may vary significantly across traders. Hence a rational expectation model (where by definition agents don't disagree on the model) can generate differential interpretation of public signals and trade. ${ }^{6}$

\section{Trading volume statistics}

\subsection{Preliminary studies}

The trading volume is defined to be the sum of the positive parts of the traders' demands, i.e., $z=$ $\Sigma_{i=1}^{n+1} y_{i}^{+}$, where $y_{i}$ is the demand (negative or positive) of agent $i$ for the asset, and $y_{i}^{+}=I\left[y_{i}>0\right] y_{i}$, with $I$ being the indicator function. As $z$ is the sum of the truncated demand $y_{i}$ 's, the statistics characterizing $z$ depend not only on the characteristics of every individual $y_{i}$, but also on the crosscorrelations, which depends in turn in very non-linear ways on the cross-correlations of the informed traders' signals, that is, on how much they agree. The traders' demands are cross-correlated even if the original signals are not for two reasons. First each individual equilibrium valuation depend on all the other valuations, this might induce correlation across them. Second, the demands depend on price, which is a weighted average of traders' valuations. Besides, as proposition (2) shows, the demand of a well-informed trader will have the same coefficient of variation, skewness, and kurtosis as the demand of a non-informed one. This is because these measures are normalized. 7

Proposition 2 The coefficient of variation, skewness and kurtosis of the each informed trader's demand are independent of the precision of the informed trader's signal and the correlation between

\footnotetext{
${ }^{6}$ A different approach is taken by Harris and Raviv (1993), and Kandel and Pearson (1995).

${ }^{7}$ Recall that, for a random variable $\omega$, the coefficient of variation is $c(\omega)=\frac{\sigma_{\omega}}{E[\omega]}$, the skewness is $s(\omega)=\frac{E\left[(\omega-E[\omega])^{3}\right]}{\operatorname{var}(\omega)^{\frac{3}{2}}}$, and the kurtosis is $k(\omega)=\frac{E\left[(\omega-E[\omega])^{4}\right]}{\operatorname{var}(\omega)^{2}}$.
} 
these signals, and are equal to the coefficient of variation, skewness and kurtosis of a standard normal random variable truncated at zero.

The proof is in the appendix.

The dependence of the trading volume's normalized statistics on the correlations between the demand is made clear in the case of one liquidity trader and two informed traders, as shown in proposition (3).

Proposition 3 With two informed traders and one liquidity trader the coefficient of variation, the skewness and the kurtosis of the aggregate volume depend only on the correlation between the demands of the two informed traders, and the ratio of the variances of the demands

The proof is in the appendix.

An important consequence of proposition (3) is that, if the informed traders have identically precise information (i.e., $\rho_{1}=\rho_{2}$ ), the coefficient of variation, the skewness and the kurtosis of the aggregate trading volume depend only on the how they agree, i.e., on the correlation between the two initial signals. Proposition (3) also shows that the coefficient of variation, the skewness or the kurtosis cannot be used to detect differences between the overall information quality of two markets when the quality of the private information is equal across traders within each market. Those statistics will be the same in a market with identically poorly informed traders and in one with identically well informed traders.

With the coefficient of variation, skewness and the kurtosis, in a model with two informed traders and one liquidity trader, are plotted on Figure (3) as functions of the ratio of the variance of the informed traders and the correlation between their demands. Without loss of generality, the demand variance ratio can be constrained to be between zero and one ${ }^{8}$ When one trader has no information, the demand variance ratio is zero, when both traders are equally well informed, the ratio is one. When the ratio decreases, the coefficient of variation tends to go up, but the skewness and the kurtosis go up or down, depending on the correlation between the demand. Besides, for any given value of the variance ratio, the effect of increasing the correlation between the traders' demands is not monotonic. Ultimately, the non-monotonicities in the relation between demands' characteristics (variance ratio and correlation) and volume characteristics are compounded by the complexity of the relations between the characteristics of the traders' information and those of their demands. Still, the graphs reveal some characteristics of volume which are independent of the demand covariance structure. Aggregate volume exhibits positive skewness and excess kurtosis (i.e., kurtosis superior to 3 , the value for a normally distributed variable). Its mean is superior

\footnotetext{
${ }^{8}$ If the ratio were superior to one, one would relabel the two agents and invert the ratio.
} 
to its median and the tail of its distribution is fatter than for a normal distribution. In sum, its distribution is dominated by rare occurrence of large surges in volume.

\section{$2.2 \quad$ Numerical examples}

The fundamental parameters are: the correlations between the signals and true asset value $\left(\rho_{1}\right.$ and $\left.\rho_{2}\right)$, the correlation between these signals $(\gamma)$, and the variance of the liquidity shock $\left(\sigma_{\varepsilon}^{2}\right)$. The final objects of interest are the following characteristics of the equilibrium price and aggregate volume: the variance of the price, its correlation with the asset value (its 'informativeness'), the mean and variance of the aggregate volume, its coefficient of variation, skewness and kurtosis. By construction, the mean price is zero. The relation between the fundamentals and the final object of analysis are complex, and few conclusions can be derived analytically. Therefore, I resort to simulations, varying some parameters and trying to make sense of the effect on the price and the volume. 9 The informed traders' signals interact with the liquidity shock at two stages: the formation of the equilibrium valuations, and the formation of the equilibrium volume. This complex mixing of the traders' information may explain the non-linearities in the relations between the fundamentals on one side, and the price and the aggregate volume on the other. One result that can be derived analytically is that, with a liquidity trader and a single informed trader, the equilibrium price variance, its informativeness, the mean and variance of the equilibrium volume are increasing in the precision of the informed trader's signal. As shown below, these results are still valid with multiple informed traders.

I construct four experiments. As the effect of varying one parameter might depend on the value of all the others, for each experiment, I vary two parameters, and produce a three-dimensional graph and a contour graph, where a darker shade indicates lower values for the variable. In the first experiment, the precision of the signal is identical across traders $\left(\rho_{1}=\rho_{2}=\rho\right.$ ), I vary the common level of the precision and the correlation of the signals $(\gamma)$. In the second experiment, the precision of the second signal is maintained constant at 0.5 , while I vary the precision of the first signal and the correlation between the two signals. In the third experiment, the correlation among signals is set equal to zero, and I vary the precision of each signal independently. In the fourth experiment, the precision of the signal is identical across traders, I vary the common level of the precision and the variance of the liquidity shock. In the following, I describe the main features of the graphs and try to give some intuitive explanations for them. Furthermore, results are summarized in table (2) For each experiment, I estimate the semi-elasticities of some observable market statistics-the price variance, the volume mean and variance-with respect to the parameters

\footnotetext{
${ }^{9}$ To better understand the simulations' results, recall that if $\omega$ is a normally distributed random variable, and $\omega^{+}$ is the positive truncation of $\omega$, the approximate values for $\operatorname{var}\left(\omega^{+}\right), c\left(\omega^{+}\right), s\left(\omega^{+}\right)$, and $k\left(\omega^{+}\right)$are $0.34,1.46,1.64$, and 5.41 .
} 
used in the experiment. The semi-elasticities are obtained by regressing the logarithms of the market statistics on a constant and the levels of the parameters used in the experiments.

In the first experiment, I vary the correlation between the signals and the common value of their precision. The graphs are displayed in Figures (4) to (7). Whatever the correlation between the signals is, increasing their common precision tends to raise the price variance, its informativeness, together with the volume mean and variance, while lowering the coefficient of variation. The effect on the skewness and kurtosis is less clear, as it depends on the correlation between the signals. To shed some light on the patterns for price, recall that the market-clearing price is a weighted sum of the valuations and the noise: $p=\theta_{1} v_{1}+\theta_{2} v_{2}+\left(1-\theta_{1}-\theta_{2}\right) \varepsilon$, with $\theta_{i}=\frac{\alpha_{i}}{\alpha_{1}+\alpha_{2}+b}$, and $\alpha_{i}=\frac{1}{\operatorname{var}\left(x \mid G_{i}, p\right)}$, for $i=1,2$. The more precise the original signals are, the higher are the variance of the valuations, their correlation, and their weight $\theta_{i}$. Hence, more precise signals generate a more volatile and informative market price. As for the patterns for volume, with more precise information, each trader's demand becomes more volatile, resulting in a higher and more volatile aggregate volume.

The precision of the price tends to go down as the correlation between signals increases when $\rho \geq .5$ (for lower values of $\rho$, the price informativeness as a function of $\gamma$ tends to have a U-shape that is more difficult to explain). That the price might be less informative when the signals are more correlated is quite intuitive since the market price is a noisy aggregate of the traders' information; a more diverse pool of private signals (a low correlation between signals) will generate a more informative equilibrium price. This intuition is even clearer for a totally revealing price, i.e., for $p^{*}=E\left[x \mid G_{1}, G_{2}\right]$; as the correlation coefficient between $G_{1}$ and $G_{2}$ gets smaller, the pair $\left(G_{1}, G_{2}\right)$ spans more of the space of the possible realizations of $x$ and makes the resulting equilibrium price $p^{*}$ more informative. Of course, in our case, the market-clearing price is a noisy aggregate of the signals, but the intuition should hold.

The variance of the volume is increasing with the correlation of the signals, while the mean volume is decreasing in this parameter. The effect of increasing the correlation of the signals on the coefficient of variation, the skewness and the kurtosis of the volume depends on the level of the information precision. The last three statistics attain their minimum when the informed traders' signals are very precise and not correlated. This shows that informed trading does not always translate into fat tails in the distribution of volume.

In the second experiment, the precision of the second signal is maintained constant at 0.5 , while I vary the precision of the first signal and the correlation between signals. Graphs are displayed in Figures (8) and (11). Whatever the correlation between signals is, increasing the precision of one signal raises the variance of the price and its correlation with the true value of the asset. The mean and variance of the volume tend also to increase. The coefficient of variation, 
the skewness and the kurtosis linger inside a narrow band. As in case 1, they are the lowest when the precision of the signals is high and their correlation low.

In the third experiment, I increase the quality of the two traders' signals independently, while maintaining at zero the correlation between signals. Graphs are displayed in Figures (12) and (15). Whatever the precision of one trader's signal is, increasing the precision of the other trader's signal increases the variance of the price, its correlation with the true value of the asset, and the volume mean and variance. The coefficient of variation, the skewness and the kurtosis display non-linear patterns while varying little. Notice that those variables attain a valley when the precision of both signals precision are the highest.

In the fourth experiment, I vary the variance of the liquidity shock and the common information precision of the traders. The correlation of their signals is kept fixed at zero. Graphs are displayed in Figures (16) and (19). Recall that, the way the liquidity demand has been constructed, without informed traders, the variance of the market-clearing price would be equal to the variance of the liquidity shock $\sigma_{\varepsilon}^{2}$. From the onset, the distribution of the true value of the asset was set equal to the distribution of the market-clearing price with no informed trading. As the variance of the true value of the asset is fixed to unity, 1 represents the "reference" level for the liquidity shock variance, which gives an idea of the range of $\sigma_{\varepsilon}^{2}$ used in the current experiment. Increasing the liquidity variance raises the mean and variance of the volume while increasing the variance of the price and decreasing its informativeness. Notice that the effect of a higher liquidity shock variance on the observable characteristics of price and volume are qualitatively the same as for an increase in the traders' information precision, while the effect on the price informativeness is flipped around. An increase in the informativeness of the private signals may be distinguished from an increase in the liquidity variance via the size of the effect on the observable characteristics of price and volume. Making the liquidity noise more volatile increase the variance of the volume much more than its mean, whereas increasing the precision of the informed traders' signals has roughly the same effect on both variables. However, in the current example the slope of the liquidity demand is set equal to one, results could be sensitive to this parameter. 


\section{Conclusion}

The paper relates patterns of the market price and the trading volume to the distribution of information across traders. Through numerical examples, it is shown that, when traders have better information, the mean and variance of the trading volume and the variance of the market price tend to be higher, and that the market price naturally becomes more informative. The effect of an increase in the liquidity shock variance is similar (though smaller), except of course for the price informativeness. Varying the correlation between the informed traders' signals has a smaller effect than varying the information quality, except for the coefficient of variation, skewness and kurtosis, which are very sensitive to the correlation across the informed traders' signals. In particular, fat tails for volume (i.e., high kurtosis) do not indicate that the traders' information about the asset value is highly precise.

Increasing the correlation across the informed traders' signals (so that the traders agree more with each other) lowers the volume mean and the price variance, while increasing the volume variance. The difference in the impact of varying the correlation between the informed traders' signals and the true asset value (the precision of their information), and varying the correlation across their signals (the degree of consensus) on the observable price and volume statistics could help us distinguish between public and private information, since public information would increase the degree of consensus among traders while making them more informed, whereas private information

would affect primarily the precision of one or more informed trader's signals without increasing the degree of consensus amomg traders. 


\section{APPENDIX}

\section{A Characteristics of trading volume}

Proposition 4 Let $x$ a standard normal random variable and $x^{+}=I[x>0] x$. Let $M_{x}(t)$ be the moment generating function of $x^{+}$. Then, $M_{x}(t)=\frac{1}{2}+\exp \left(\frac{1}{2} t^{2}\right) \Phi(t)$. where $\Phi$ is the standard normal cdf. Consequently,

$$
\begin{array}{lll}
E\left[x^{+}\right] & =\frac{1}{\sqrt{2 \pi}}, & E\left[\left(x^{+}\right)^{2}\right]=\frac{1}{2} \\
E\left[\left(x^{+}\right)^{3}\right]=\frac{2}{\sqrt{2 \pi}}, & E\left[\left(x^{+}\right)^{4}\right]=\frac{3}{2}
\end{array}
$$

It follows that $c\left(x^{+}\right)=c=\sqrt{\pi-1}, s\left(x^{+}\right)=s=\frac{\frac{1}{\sqrt{2 \pi}}\left(\frac{1}{\pi}+\frac{1}{2}\right)}{\left(\frac{1}{2}-\frac{1}{2 \pi}\right)^{3 / 2}}$, and $k\left(x^{+}\right)=k=\frac{\frac{1}{2}\left(3-\frac{5}{\pi}-\frac{3}{2 \pi^{2}}\right)}{\left(\frac{1}{2}-\frac{1}{2 \pi}\right)^{2}}$.

Proof of proposition 4: By definition, $M_{x}(t)=E\left[e^{t x^{+}}\right]=p(x \leq 0)+E\left[I[x>0] e^{x t}\right]$.

$$
\begin{aligned}
E\left[I[x>0] e^{x t}\right] & =\int_{x=0}^{+\infty} e^{x t} \phi(x) d x \\
& =\int_{x=0}^{+\infty} \frac{1}{\sqrt{2 \pi}} e^{x t} e^{-\frac{1}{2} x^{2}} d x \\
& =\int_{x=0}^{+\infty} \frac{1}{\sqrt{2 \pi}} e^{-\frac{1}{2}\left[(x-t)^{2}-t^{2}\right]} d x \\
& =e^{\frac{1}{2} t^{2}} \int_{u=-\infty}^{t} \frac{1}{\sqrt{2 \pi}} e^{-\frac{1}{2} u^{2}} d u
\end{aligned}
$$

Results in equation (16) can be obtained by taking derivatives of the moment generating function, then proposition (4) follows straightforwardly.

Proof of proposition 2: Write $y_{i}$ the demand of the $i^{t h}$ informed trader, $\tilde{y}_{i}=\frac{1}{\sigma_{i}} y_{i}$, where $\sigma_{i}^{2}=\operatorname{var}\left(y_{i}\right)$. Proposition (2) follows from the fact that $\tilde{y}_{i}$ is a standard normal, and proposition (4).

Proposition (2) shows that the characteristics of the traded volume will be in fact determined by the interactions between the individual demand schedules, in particular their covariances. Indeed, a sum of independently and identically distributed random variables have the same coefficient of variation, skewness and kurtosis as each random variable in the sum.

Now, we use the fact that $y_{1}+y_{2}+y_{3}=0$. Let $\rho_{i j}=\operatorname{cov}\left(y_{i}, y_{j}\right), \rho=\rho_{12}, \sigma_{i}^{2}=\operatorname{var}\left(y_{i}\right)$, and $\sigma_{i j}=\operatorname{cov}\left(y_{i}, y_{j}\right), s=\frac{\sigma_{1}}{\sigma_{2}}$. Using the fact that $y_{1}+y_{2}+y_{3}=0$, we get $\sigma_{3}^{2}=\sigma_{1}^{2}+\sigma_{2}^{2}+2 \sigma_{12}=$ $\sigma_{1} \sigma_{2}\left(s+\frac{1}{s}+2 \rho\right)$, and after some manipulations

$$
\begin{array}{rlll}
\sigma_{1} \sigma_{3} & =\sigma_{1} \sigma_{2} \sqrt{s^{2}+2 \rho s+1} & \sigma_{2} \sigma_{3} & =\sigma_{1} \sigma_{2} \sqrt{\frac{1}{s^{2}}}+2 \rho \frac{1}{s}+1 \\
\frac{\sigma_{1}}{\sigma_{3}} & =\sqrt{\frac{s}{s+\frac{1}{s}+\rho}} & \frac{\sigma_{1}}{\sigma_{3}} & =\sqrt{\frac{\frac{1}{s}}{s+\frac{1}{s}+\rho}} \\
\rho_{13} & =-\frac{s+\rho}{\sqrt{s^{2}+2 \rho s+1}} & \rho_{23} & =-\frac{\frac{1}{s}+\rho}{\sqrt{\frac{1}{s^{2}}+2 \rho \frac{1}{s}+1}}
\end{array}
$$

Proof of proposition 3: We begin by the skewness and the kurtosis. Let $\tilde{y}_{i}=\frac{1}{\sigma_{i}} y_{i}, z_{i}=$ $y_{i}^{+}-E\left[y_{i}^{+}\right], \tilde{z}_{i}=\tilde{y}_{i}^{+}-E\left[\tilde{y}_{i}^{+}\right]$, and $\mu=E[z]$, then, $z-\mu=z_{1}+z_{2}+z_{3} . E\left[(z-\mu)^{n}\right]$ is a weighted 
sum of $E\left[z_{1}^{a_{1}} z_{2}^{a_{2}} z_{3}^{a_{3}}\right]$, with $a_{1}+a_{2}+a_{3}=n$, while $a_{i}$ is an integer in $[0, n]$.

$$
\begin{aligned}
E\left[z_{1}^{a_{1}} z_{2}^{a_{2}} z_{3}^{a_{3}}\right] & =\sigma_{1}^{a_{1}} \sigma_{2}^{a_{2}} \sigma_{3}^{a_{3}} E\left[\tilde{z}_{1}^{a_{1}} \tilde{z}_{2}^{a_{2}} \tilde{z}_{3}^{a_{3}}\right] \\
& =\sigma_{1}^{a_{1}} \sigma_{2}^{a_{2}} \sigma_{3}^{a_{3}} g(\rho, s) \\
& =\left(\frac{\sigma_{1}}{\sigma_{3}}\right)^{a_{1}}\left(\frac{\sigma_{2}}{\sigma_{3}}\right)^{a_{2}} \sigma_{3}^{n} g(\rho, s) \\
& =\left[\sqrt{\sigma_{1} \sigma_{2}}\right]^{n} f(s, \rho)
\end{aligned}
$$

The second line comes from the fact that cross moments of normal truncated variables are functions of the covariance matrix of the underlying normal variables. More precisely $\tilde{y}_{3}=-\frac{\sigma_{1}}{\sigma_{3}} \tilde{y}_{1}-\frac{\sigma_{2}}{\sigma_{3}} \tilde{y}_{2} \cdot \frac{\sigma_{1}}{\sigma_{3}}$, and $\frac{\sigma_{2}}{\sigma_{3}}$ depend on $\rho$, and $s$, and $\left(\tilde{y}_{1}, \tilde{y}_{2}\right)$ is completely determined by rho. Hence $E\left[z_{1}^{a_{1}} z_{2}^{a_{2}} z_{3}^{a_{3}}\right]$ is a function of only two parameters: $\rho$, and $s$. In the third line, we use the fact that $a_{1}+a_{2}+a_{3}=n$. The fourth line was obtained using (18). Consequently, $E\left[(z-\mu)^{n}\right]=\left[\sqrt{\sigma_{1} \sigma_{2}}\right]^{n} \lambda(s, \rho)$. The $\sigma_{1} \sigma_{2}$ terms cancel when we compute skewness and kurtosis. As one can always permute the indices of the two informed traders, we need only $s$ between zero and one, in other words, if $c(\rho, s)$ is the value of the coefficient of variation, $c(\rho, s)=c\left(\rho, \frac{1}{s}\right)$. The same remark applies for the skewness and the kurtosis.

\section{B Computation of volume's statistics}

Table (1) gives the definition of $z$ in terms of $y_{1}, y_{2}$, and $y_{3}$ in the six different cases. $z=\Sigma_{i=1}^{6} I\left[v_{1}^{i}>\right.$ $\left.0, v_{2}^{i}>0\right]\left(v_{1}^{i}+v_{2}^{i}\right)$, with $\left(v_{1}^{1}, v_{2}^{1}\right)=\left(y_{1}, y_{2}\right),\left(v_{1}^{2}, v_{2}^{2}\right)=\left(-y_{2}, y_{1}+y_{2}\right),\left(v_{1}^{3}, v_{2}^{3}\right)=\left(y_{1},-\left(y_{1}+y_{2}\right)\right)$, $\left(v_{1}^{4}, v_{2}^{4}\right)=\left(-y_{1}, y_{1}+y_{2}\right),\left(v_{1}^{5}, v_{2}^{5}\right)=\left(y_{2},-\left(y_{1}+y_{2}\right)\right),\left(v_{1}^{6}, v_{2}^{6}\right)=\left(-y_{1},-y_{2}\right)$. Hence, to compute the various moments of $z$, one need only calculate $E\left[I\left[v_{1}^{i}>0, v_{2}^{i}>0\right]\left(v_{1}^{i}+v_{3}^{i}\right)\right]$, for $i=1, \ldots, 4$.

B.1 Computing $E\left[I\left[v_{1}>0, v_{2}>0\right]\right.$.

\section{Proposition 5}

$$
E\left[I\left(v_{1}>0\right) I\left(v_{2}>0\right)\left(v_{1}+v_{2}\right)\right]=\frac{\sigma_{1}^{v}+\sigma_{2}^{v}}{2 \sqrt{2 \pi}}\left(1+\rho_{12}^{v}\right)
$$

proof: Let $f$ be the density function of the normally distributed vector $\left(v_{1}, v_{2}\right)^{\prime}$ with mean zero and variance $\Sigma$. Note $\Sigma=\left(\sigma_{i j}\right)_{i, j=1}^{2}$, and $\Sigma^{-1}=\left(\sigma^{i j}\right)_{i, j=1}^{2} . \quad f(v)=\frac{1}{2 \pi} \operatorname{det}(\Sigma)^{-\frac{1}{2}} e^{-\frac{1}{2} v^{\prime} \Sigma^{-1} v}$ Let $\nabla f=\left(f_{1}\left(v_{1}, v_{2}\right), f_{2}\left(v_{1}, v_{2}\right)\right)^{\prime}=-\Sigma^{-1} v f(v)$, which implies the following.

$$
\left\{\begin{array}{l}
f_{1}\left(v_{1}, v_{2}\right)=-\left\{\sigma^{11} v_{1}+\sigma^{12} v_{2}\right\} f\left(v_{1}, v_{2}\right) \\
f_{2}\left(v_{1}, v_{2}\right)=-\left\{\sigma^{21} v_{1}+\sigma^{22} v_{2}\right\} f\left(v_{1}, v_{2}\right)
\end{array}\right.
$$

For $i=1,2$, we have $E\left[I\left(v_{1}<0, v_{2}<0\right) v_{i}\right]=\int_{-\infty}^{0} \int_{-\infty}^{0} v_{i} f\left(v_{1}, v_{2}\right) d v_{1} d v_{2}$. 
Note $I=I\left[v_{1}>0, v_{2}>0\right]$,

$$
\begin{aligned}
\sigma^{11} E\left[I y_{1}\right]+\sigma^{12} E\left[I y_{2}\right] & =\int_{-\infty}^{0} \int_{-\infty}^{0}\left[\sigma^{11} v_{1}+\sigma^{12} v_{2}\right] f\left(v_{1}, v_{2}\right) d v_{1} d v_{2} \\
& =-\int_{v_{2}=-\infty}^{v_{2}=0}\left[f\left(v_{1}, v_{2}\right)\right]_{v_{1}=-\infty}^{v_{1}=0} d v_{2} \\
& =-\int_{-\infty}^{0} \frac{1}{2 \pi \sqrt{|\Sigma|}} e^{-\frac{1}{2} \sigma^{22} t^{2}} d t \\
& =-\frac{1}{\sqrt{2 \pi|\Sigma| \sigma^{22}}} \int_{-\infty}^{0} \frac{1}{\sqrt{2 \pi}} e^{-\frac{1}{2} u^{2}} d u \\
& =-\frac{1}{\sqrt{2 \pi|\Sigma| \sigma^{22}}} \Phi(0) \\
& =-\frac{1}{2 \sigma_{1} \sqrt{2 \pi}}=c_{1}
\end{aligned}
$$

In the fourth line, the change of variable $u=\sqrt{\sigma^{22}} t$ was used. For the last but one equation, by definition, $\sigma^{22}=\frac{\sigma_{11}}{|\Sigma|}$, and $\sigma_{1}=\sqrt{\sigma_{11}}$.

In the same fashion

$$
\sigma^{11} E\left[I y_{1}\right]+\sigma^{12} E\left[I y_{2}\right]=-\frac{1}{2} \frac{1}{\sigma_{2} \sqrt{2 \pi}}=c_{2}
$$

Equations (22), and (23) can be rewritten as $\Sigma^{-1}\left(E\left[I y_{1}\right], E\left[I y_{2}\right]\right)^{\prime}=c$, with $c=\left(c_{1}, c_{2}\right)^{\prime}$. Hence, $\left(E\left[I y_{1}\right], E\left[I y_{2}\right]\right)^{\prime}=\Sigma c$ and

$$
E\left[I\left(v_{1}<0, v_{2}<0\right) v_{i}\right]=-\frac{\sigma_{i}}{2 \sqrt{2 \pi}}\left[1+\rho_{12}\right],
$$

where $\rho_{12}$ is the correlation coefficient between $v_{1}$ and $v_{2}$. Also, $p\left(v_{1}<0, v_{2}<0\right)=\frac{1}{4}+$ $\frac{1}{2 \pi} \arcsin \left(\rho_{12}\right)$.

\section{B.2 Computing $E\left[I\left[v_{1}>0, v_{2}>0\right]\left(v_{1}+v_{2}\right)^{2}\right]$}

Let $G^{1}\left(v_{1}, v_{2}\right)=v_{1} f\left(v_{1}, v_{2}\right)$, where $f$ is the density function of $v$. Let $G_{i}^{1}=\frac{\partial G^{1}}{\partial v_{i}}, i=1,2$. Then $G_{1}^{1}=v_{1} f_{1}+f$, and $G_{2}^{1}=v_{1} f_{2}$. Hence,

$$
\begin{aligned}
& \left(\sigma^{11} v_{1}^{2}+\sigma^{12} v_{1} v_{2}\right) f=-v_{1} f_{1}=f-G_{1}^{1} \\
& \left(\sigma^{12} v_{1}^{2}+\sigma^{22} v_{1} v_{2}\right) f=-v_{1} f_{2}=-G_{2}^{1}
\end{aligned}
$$

We will write $v=\left(v_{1}, v_{2}\right), I=I\left[v_{1}>0, v_{2}>0\right]$, and $p(v>0)=p\left(v_{1}>0, v_{2}>0\right)$. Noting that, for any value of $v_{2}, \int_{v_{1}=0}^{\infty} G_{1}^{1}\left(v_{1}, v_{2}\right) d v_{1}=\left[G^{1}\left(v_{1}, v_{2}\right)\right]_{v_{1}=0}^{\infty}=0$, we get

$$
\sigma^{11} E\left[I v_{1}^{2}\right]+\sigma^{12} E\left[I v_{1} v_{2}\right]=p(v>0)
$$


Also,

$$
\begin{aligned}
\sigma^{12} E\left[I v_{1}^{2}\right]+\sigma^{22} E\left[I v_{1} v_{2}\right] & =-\int_{v_{1}=0}^{\infty} \int_{v_{2}=0}^{\infty} \frac{\partial}{\partial v_{2}} G^{1}\left(v_{1}, v_{2}\right) d v_{1} d v_{2} \\
& =-\int_{v_{1}=0}^{\infty}\left[G^{1}\left(v_{1}, v_{2}\right)\right]_{v_{2}=0}^{\infty} d v_{1} \\
& =\frac{1}{2 \pi \sqrt{|\Sigma|}} \int_{v_{1}=0}^{\infty} v_{1} e^{-\frac{1}{2} \sigma^{11} v_{1}^{2}} d v_{1} \\
& =\frac{1}{2 \pi \sigma^{11} \sqrt{|\Sigma|}} \int_{u=0}^{\infty} u e^{-\frac{1}{2} u^{2}} d u \\
& =\frac{1}{2 \pi \sigma^{11} \sqrt{|\Sigma|}} \\
& =\frac{\sqrt{|\Sigma|}}{2 \pi \sigma_{22}}
\end{aligned}
$$

Note that $\frac{1}{\sigma^{11} \sqrt{|\Sigma|}}=\frac{\sqrt{|\Sigma|}}{\sigma_{22}}$, as, by definition $\sigma^{11}=\frac{\sigma_{22}}{|\Sigma|}$. Hence,

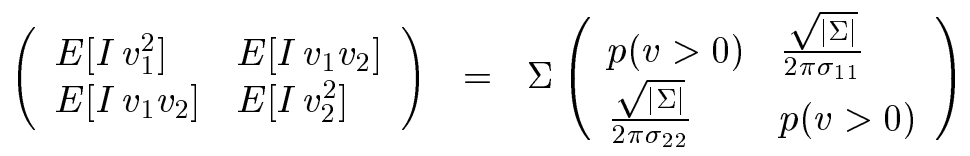

We can simplify notations, using $|\Sigma|=\sigma_{1}^{2} \sigma_{2}^{2}-\sigma_{12}^{2}$, which yields $\frac{\sqrt{|\Sigma|}}{\sigma_{22}}=\frac{\sigma_{1}}{\sigma_{2}} \sqrt{1-\rho_{12}^{2}}$. We conclude that

$$
\begin{aligned}
& E\left[I\left[v_{1}>0, v_{2}>0\right] v_{1}^{2}\right]=\sigma_{1}^{2}\left\{\frac{1}{4}+\frac{1}{2 \pi} \arcsin \left(\rho_{12}\right)+\frac{1}{2 \pi} \rho_{12} \sqrt{1-\rho_{12}^{2}}\right\} \\
& E\left[I\left[v_{1}>0, v_{2}>0\right] v_{1} v_{2}\right]=\sigma_{12}\left(\frac{1}{4}+\frac{1}{2 \pi} \arcsin \left(\rho_{12}\right)\right)+\frac{1}{2 \pi} \sigma_{1} \sigma_{2} \sqrt{1-\rho_{12}^{2}}
\end{aligned}
$$

\section{B.3 Computing $E\left[I\left[v_{1}>0, v_{2}>0\right]\left(v_{1}+v_{2}\right)^{3}\right]$}

We compute $E\left[I v_{1}^{3}\right], E\left[I v_{1}^{2} v_{2}\right], E\left[I v_{1} v_{2}^{2}\right], E\left[I v_{2}^{3}\right]$. Let $G^{1}\left(v_{1}, v_{2}\right)=v_{1}^{2} f\left(v_{1}, v_{2}\right)$, then $G_{1}^{1}(v)=$ $2 v_{1} f(v)+v_{1}^{2} f_{1}(v), G_{2}^{1}(v)=v_{1}^{2} f_{2}(v)$.

$$
\begin{aligned}
& \sigma^{11} v_{1}^{3}+\sigma^{12} v_{1}^{2} v_{2}=-v_{1}^{2} f_{1}\left(v_{1}, v_{2}\right)=2 v_{1} f(v)-G_{1}^{1}(v) \\
& \sigma^{12} v_{1}^{3}+\sigma^{22} v_{1}^{2} v_{2}=-v_{1}^{2} f_{2}\left(v_{1}, v_{2}\right)=-G_{2}^{1}(v)
\end{aligned}
$$

Noting that, for any value of $v_{2}, \int_{v_{1}=0}^{\infty} G_{1}^{1}\left(v_{1}, v_{2}\right) d v_{1}=0$, we get $\sigma^{11} E\left[I v_{1}^{3}\right]+\sigma^{12} E\left[I v_{1}^{2} v_{2}\right]=$ $2 E\left[I v_{1}\right]$. Also,

$$
\begin{aligned}
\sigma^{12} E\left[I v_{1}^{3}\right]+\sigma^{22} E\left[I v_{1}^{2} v_{2}\right] & =-\int_{v_{1}=0}^{\infty} \int_{v_{2}=0}^{\infty} G_{2}^{1}\left(v_{1}, v_{2}\right) d v_{1} d v_{2} \\
& =-\int_{v_{1}=0}^{\infty}\left[G^{1}\left(v_{1}, v_{2}\right)\right]_{v_{2}=0}^{\infty} d v_{1} \\
& =\frac{1}{2 \pi \sqrt{|\Sigma|}} \int_{v_{1}=0}^{\infty} v_{1}^{2} e^{-\frac{1}{2} \sigma^{11} v_{1}^{2}} d v_{1} \\
& =\frac{1}{2 \pi \sqrt{|\Sigma|}\left(\sigma^{11}\right)^{\frac{3}{2}}} \int_{0}^{\infty} u^{2} e^{-\frac{1}{2} u^{2}} d v_{1} \\
& =\frac{1}{2 \sqrt{2 \pi}} \frac{|\Sigma|}{\left(\sigma_{22}\right)^{\frac{3}{2}}}
\end{aligned}
$$


We used the change of variable $u=\sqrt{\sigma^{11}} v_{1}$, the identity $\sigma^{11}=\frac{\sigma_{22}}{|\Sigma|}$, and the fact that $\int_{0}^{\infty} u^{2} e^{-\frac{1}{2} u} d u=\frac{\sqrt{2 \pi}}{2}$. Hence,

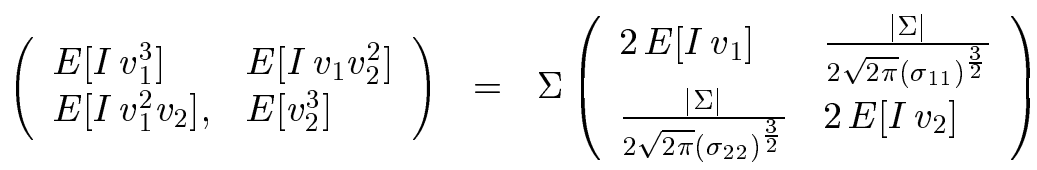

\section{B.4 Computing $E\left[I\left[v_{1}>0, v_{2}>0\right]\left(v_{1}+v_{2}\right)^{4}\right]$}

We compute $E\left[I v_{1}^{4}\right], E\left[I v_{1}^{3} v_{2}\right], E\left[I v_{1}^{2} v_{2}^{2}\right]$, etc. Let $G^{1}\left(v_{1}, v_{2}\right)=v_{1}^{3} f\left(v_{1}, v_{2}\right) . G_{1}^{1}(v)=3 v_{1}^{2} f(v)+$ $v_{1}^{3} f_{1}(v), G_{2}^{1}(v)=v_{1}^{3} f_{2}(v)$.

$$
\begin{aligned}
& \sigma^{11} v_{1}^{4}+\sigma^{12} v_{1}^{3} v_{2}=-v_{1}^{3} f_{1}\left(v_{1}, v_{2}\right)=3 v_{1}^{2} f(v)-G_{1}^{1}(v) \\
& \sigma^{12} v_{1}^{4}+\sigma^{22} v_{1}^{3} v_{2}=-v_{1}^{3} f_{2}\left(v_{1}, v_{2}\right)=-G_{2}^{1}(v)
\end{aligned}
$$

It is easy to see that $\sigma^{11} E\left[I v_{1}^{4}\right]+\sigma^{12} E\left[I v_{1}^{3} v_{2}\right]=3 E\left[I v_{1}^{2}\right]$. Also,

$$
\begin{aligned}
\sigma^{12} E\left[I v_{1}^{4}\right]+\sigma^{22} E\left[I v_{1}^{3} v_{2}\right] & =-\int_{v_{1}=0}^{\infty} \int_{v_{2}=0}^{\infty} G_{2}^{1}\left(v_{1}, v_{2}\right) d v_{1} d v_{2} \\
& =-\int_{v_{1}=0}^{\infty}\left[G^{1}\left(v_{1}, v_{2}\right)\right]_{v_{2}=0}^{\infty} d v_{1} \\
& =\frac{1}{2 \pi, \sqrt{|\Sigma|}} \int_{v_{1}=0}^{\infty} v_{1}^{3} e^{-\frac{1}{2} \sigma^{11} v_{1}^{2}} d v_{1} \\
& =\frac{1}{2 \pi \sqrt{|\Sigma|}\left(\sigma^{11}\right)^{2}} \int_{0}^{\infty} u^{3} e^{-\frac{1}{2} u^{2}} d v_{1} \\
& =\frac{1}{\pi} \frac{|\Sigma|^{\frac{3}{2}}}{\left(\sigma_{22}\right)^{2}}
\end{aligned}
$$

Hence,

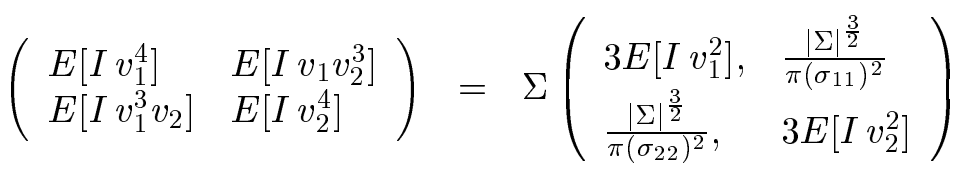

To compute $E\left[I v_{1}^{2} v_{2}^{2}\right]$, let $G^{1}\left(v_{1}, v_{2}\right)=v_{1} v_{2}^{2} f\left(v_{1}, v_{2}\right)$. Then, $G_{1}^{1}(v)=v_{2}^{2} f(v)+v_{1} v_{2}^{2} f_{1}(v)$, $G_{2}^{1}(v)=v_{1} v_{2}^{2} f_{2}(v)+2 v_{1} v_{2} f(v)$.

$$
\begin{aligned}
& \sigma^{11} v_{1}^{2} v_{2}^{2}+\sigma^{12} v_{1} v_{2}^{3}=-v_{1} v_{2}^{2} f_{1}\left(v_{1}, v_{2}\right)=v_{2}^{2} f(v)-G_{1}^{1}(v) \\
& \sigma^{12} v_{1}^{2} v_{2}^{2}+\sigma^{22} v_{1} v_{1}^{3}=-v_{1} v_{2}^{2} f_{2}\left(v_{1}, v_{2}\right)=2 v_{1} v_{2} f(v)-G_{2}^{1}(v)
\end{aligned}
$$

For any value of $v_{2}, \int_{v_{1}=0}^{\infty} G_{1}^{1}\left(v_{1}, v_{2}\right) d v_{1}=0$, and for any $v_{1}, \int_{v_{2}=0}^{\infty} G_{2}^{1}\left(v_{1}, v_{2}\right) d v_{2}=0$, hence,

$$
\begin{aligned}
\sigma^{11} E[I & \left.v_{1}^{2} v_{2}^{2}\right]+\sigma^{12} E\left[I v_{1} v_{2}^{3}\right]=E\left[I v_{2}^{2}\right] \\
\sigma^{12} E[I & \left.v_{1}^{2} v_{2}^{2}\right]+\sigma^{22} E\left[I v_{1} v_{2}^{2}\right]=2 E\left[I v_{1} v_{2}\right]
\end{aligned}
$$

Finally,

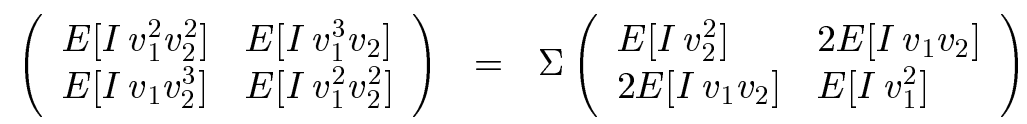




\section{REFERENCES}

Barclay, Michael, Robert H. Litzenberger, and Jerold Warner (1990), "Private Information, Trading Volume, and Stock-Return Variances", The Review of Financial Studies, Vol 3, No 2, p. 233-253.

Blume Lawrence, David Easley, and Maureen O'Hara (1994), "Market Statistics and Technical Analysis: The Role of Volume", The Journal of Finance, Vol XLIX, No 1, March 1994.

Brown, David, and Robert H. Jennings (1989), "On Technical Analysis", The Review of Financial Studies, Vol 2, No 4, p 527-551.

Diamond, Douglas and Robert E. Verrechia (1981), "Information Aggregation in a Noisy Rational Expectation Economy", Journal of Financial Economics, vol 9, p 221-235.

Dupont, Dominique (1997), "Extracting Information from Trading Volume", working paper, Federal Reserve Board.

Easley, David and Maureen O'Hara (1987), Price, Trade Size, and Information in Securities Markets, Journal of Financial Economics, Vol 19, p. 69-90.

Easley, David, Nicholas Kiefer, and Maureen O'Hara (1996), "Cream-Skimming or Profit-Sharing? the Curious Role of Purchased Order Flow", The Journal of Finance, Vol 51, No 3, pp. 811-833.

Easley, David, Nicholas Kiefer, Maureen O'Hara, and Peperman (1996), "Liquidity, Information, and Infrequently Traded Stocks", The Journal of Finance, Vol 51, No 4, pp. 1405-1436.

Fleming, M. and E. Remola (1996), "Price Formation and Liquidity in the U.S. Treasuries Market: Evidence from Intraday Patterns around Announcements", working paper, Federal Reserve Bank of New York.

Foster, F. D., and S. Viswanathan (1995), "Can Speculative Trading Explain the Volume-Volatility Relation", Journal of Business and Economic Statistics, Vol 13, No 4, p. 379-396.

Foster, F. D., and S. Viswanathan (1996), "Strategic Trading When Agents Forecast the Forecast of Others", The Journal of Finance, Vol 51, No 4, pp. 1437-1478.

Gallant, Ronald, Peter E. Rossi, and George Tauchen (1992), "Stock Prices and Volume", The Review of Financial Studies, Vol 5, No 2, pp 199-242.

Goodman, William (1996), "Statistically Analyzing Volume", Technical Analysis of Stocks and Commodities, November 1996, p 21-28.

Grundy Bruce D., and Maureen McNichols (1990), "Trade and the Revelation of Information through Prices and Direct Disclosure", The Review of Financial Studies, Vol 2, No 4, p. 495-526.

Harris, Milton and Artur Raviv (1994), "Differences of Opinion Make a Horse Race", Review of Financial Studies, Vol 6, No 3, p 473-506. 
He, Hua and J. Wang (1995), "Differential Information and Dynamic Behavior of Stock Trading Volume", The Review of Financial Studies, Vol 8, No 4, p 919-972.

Huffman, Gregory W. (1992), "Information, Asset Prices, and the Volume of Trade", The Journal of Finance, vol XLVII, September 1992, p 1575-1590.

Jain, Prem (1988), "Responses of Hourly Stock Prices and Trading Volume to Economic News", The Journal of Business, Vol 61, No 2, pp. 219-231.

Hussman, John (1992), "Market Efficiency and Inefficiency in Rational Expectations Equilibria", Journal of Economic Dynamics and Control, p 655-680.

Kandel, E. and N. Pearson (1995), "Differential Interpretation of Public Signals and Trade in Speculative Markets", Journal of Political Economy, Vol 103, No 4, pp. 831-872.

Karpoff, Jonathan M. (1986), "A Theory of Trading Volume", Journal of Finance, vol XLI, No 5, December 1986, p 1069-1087.

Karpoff, Jonathan M. (1987), "The Relationship between Price Changes and Volume: A Survey", Journal of Financial and Quantitative Analysis, Vol 22, No 1, March 1987.

Kim, Olivier and Robert Verrechia (1991), "Market Reaction to Anticipated Announcements", Journal of Financial Economics, 30, pp. 273-309.

Kyle, Albert (1985), "Continuous Auction and Insider Trading", Econometrica, 53, p 1315-1335.

Lamoureux, Christopher G., and William D. Lastrapes (1990), "Heteroskedasticity in Stock Return Data: Volume versus GARCH effects", The Journal of Finance 12, p 31-41.

Milgrom Paul and N. Stockey (1982), "Information, Trade and Common Knowledge", Journal of Economics Theory, No 26, p. 17-27.

Saitta, Alex (1996), "A Price and Volume Based System", Technical Analysis of Stocks and Commodities, March 1996, pp 14-16.

Shalen, Catherine (1993), "Volume, Volatility, and the Dispersion of Beliefs", The Review of Financial Studies, Vol 6, no 2, p 405-434.

Wang, Jiang (1992), "A Model of Intertemporal Asset Prices under Asymmetric Information”, The Review of Financial Studies, p 249-281.

Wang, Jiang (1994), "A Model of Competitive Asset Trading Volume", Journal of Political Economy, vol 102, No 1, p 127-168. 


\begin{tabular}{|c|c|c|c|c|c|c|}
\hline cases $i$ & sign of $y_{1}$ & sign of $y_{2}$ & sign of $y_{1}+y_{2}$ & volume $z$ & $v_{1}^{i}$ & $v_{2}^{i}$ \\
\hline 1 & + & + & + & $y_{1}+y_{2}$ & $y_{1}$ & $y_{2}$ \\
\hline 2 & + & - & + & $y_{1}$ & $-y_{2}$ & $y_{1}+y_{2}$ \\
\hline 3 & + & - & - & $-y_{2}$ & $y_{1}$ & $-\left(y_{1}+y_{2}\right)$ \\
\hline 4 & - & + & + & $y_{2}$ & $-y_{1}$ & $y_{1}+y_{2}$ \\
\hline 5 & - & + & - & $-y_{1}$ & $y_{2}$ & $-\left(y_{1}+y_{2}\right)$ \\
\hline 6 & - & - & + & $-\left(y_{1}+y_{2}\right)$ & $-y_{1}$ & $-y_{2}$ \\
\hline
\end{tabular}

Table 1: Decomposition of trading volume

There are three traders; $y_{1}$ is the first trader's demand, $y_{2}$ is the second trader's demand, the last trader's demand is $y_{3}=-\left(y_{1}+y_{2}\right)$. The trading volume is $z=y_{1}^{+}+y_{2}^{+}+y_{3}^{+}$. In each case $i, i=1, \ldots, 6, z$ can be written as $v_{1}^{i}+v_{2}^{i}$. 


\begin{tabular}{|c|c|c|c|c|}
\hline & & volume mean & volume variance & price variance \\
\hline \hline case 1 & $\rho$ & 0.69 & 0.57 & 2.00 \\
$\left(\rho_{1}=\rho_{2}=\rho\right)$ & $\gamma$ & -0.07 & 0.24 & -0.22 \\
\hline case 2 & $\rho_{1}$ & 0.49 & 0.70 & 1.10 \\
$\left(\rho_{2}=0.5\right)$ & $\gamma$ & -0.09 & 0.20 & -0.19 \\
\hline case 3 & $\rho_{1}$ & 0.46 & 0.37 & 1.30 \\
$(\gamma=0)$ & $\rho_{2}$ & 0.46 & 0.37 & 1.30 \\
\hline case 4 & $\rho$ & 1.90 & 3.10 & 3.80 \\
$\left(\rho_{1}=\rho_{2}=\rho, \gamma=0\right)$ & $\sigma_{\varepsilon}^{2}$ & 0.40 & 0.87 & 0.33 \\
\hline
\end{tabular}

Table 2: Semi-elasticities of some observable price and volume statistics with respect to underlying parameters

$\rho_{1}$ is the correlation between $G_{1}$, the first trader's signal, and $x$, the true value of the asset, $\rho_{2}$ is the correlation between $G_{2}$, the second trader's signal, and $x$,

$\gamma$ is the correlation coefficient between the signals $G_{1}$ and $G_{2}$,

$\sigma_{\varepsilon}^{2}$ is the variance of the liquidity shock. 


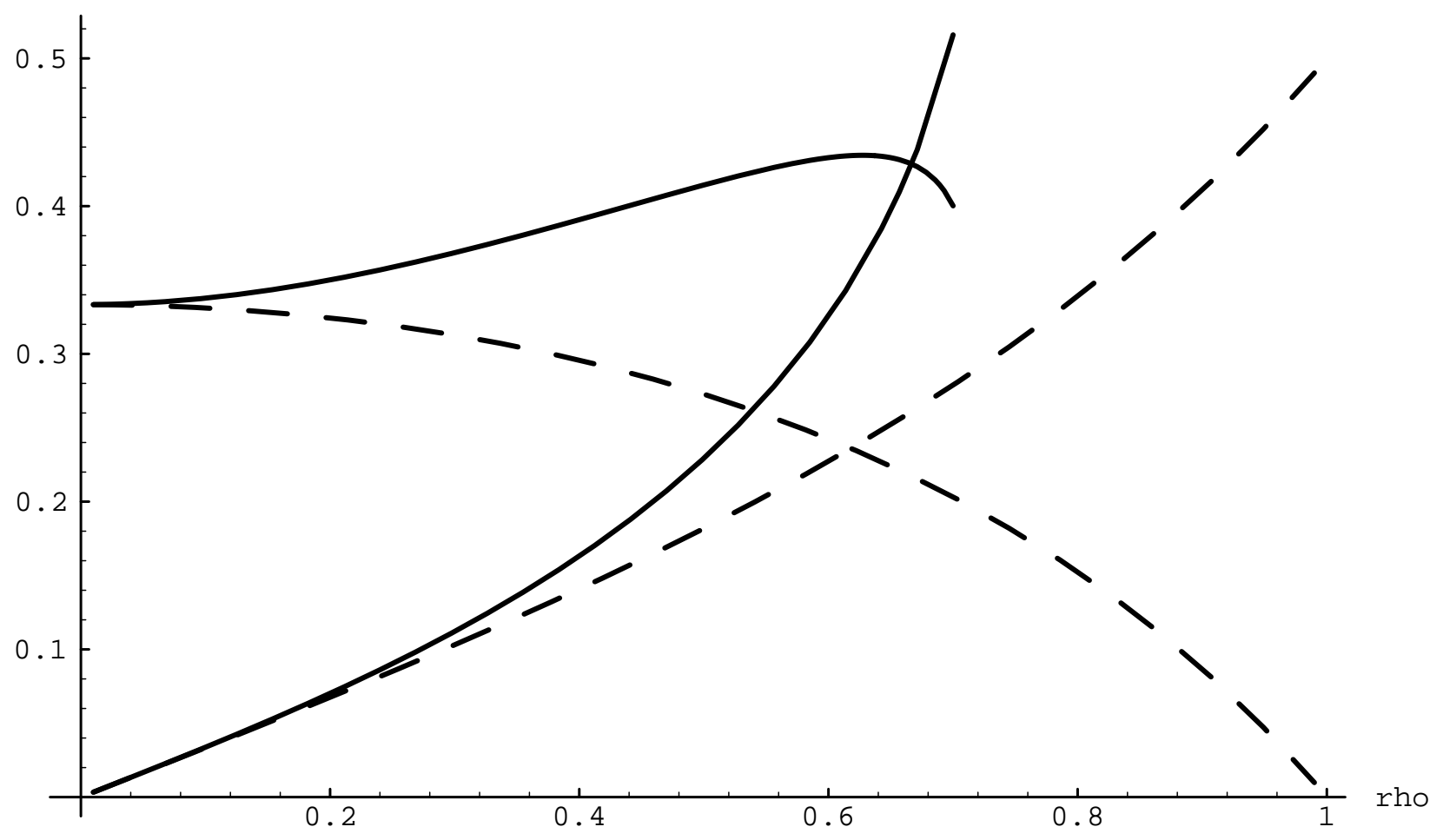

Figure 1: $p=a_{1} G_{1}+a_{2} G_{2}+a_{3} \varepsilon$, with $E\left[x G_{1}\right]=E\left[x G_{2}\right]$ and in naive traders case. 


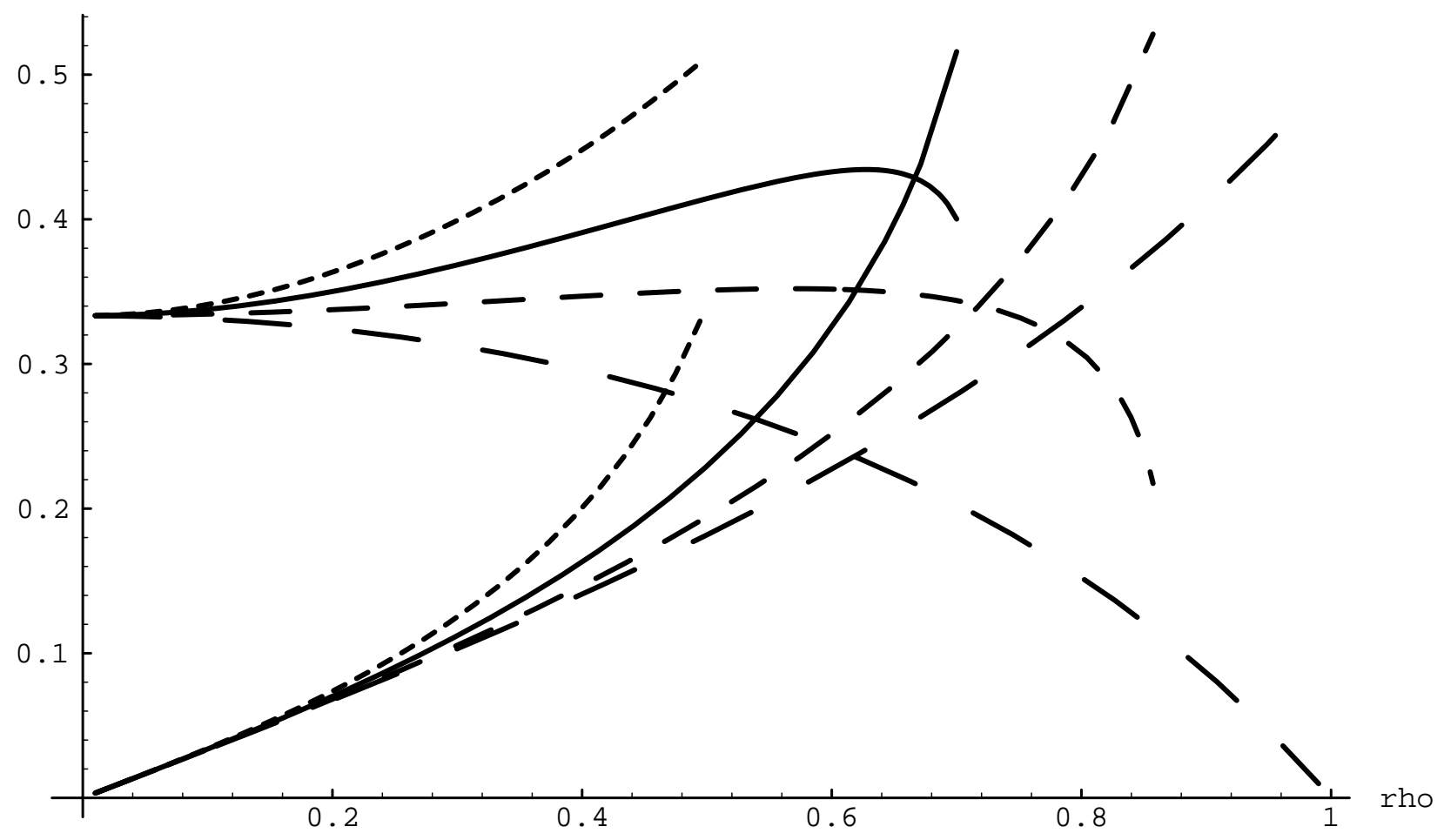

Figure 2: $p=a_{1} G_{1}+a_{2} G_{2}+a_{3} \varepsilon$ for $\gamma=-.5$ (the upper dotted lines), 0 (the solid lines), .5 (the middle dashed lines) and 1 (the lower dashed lines). 

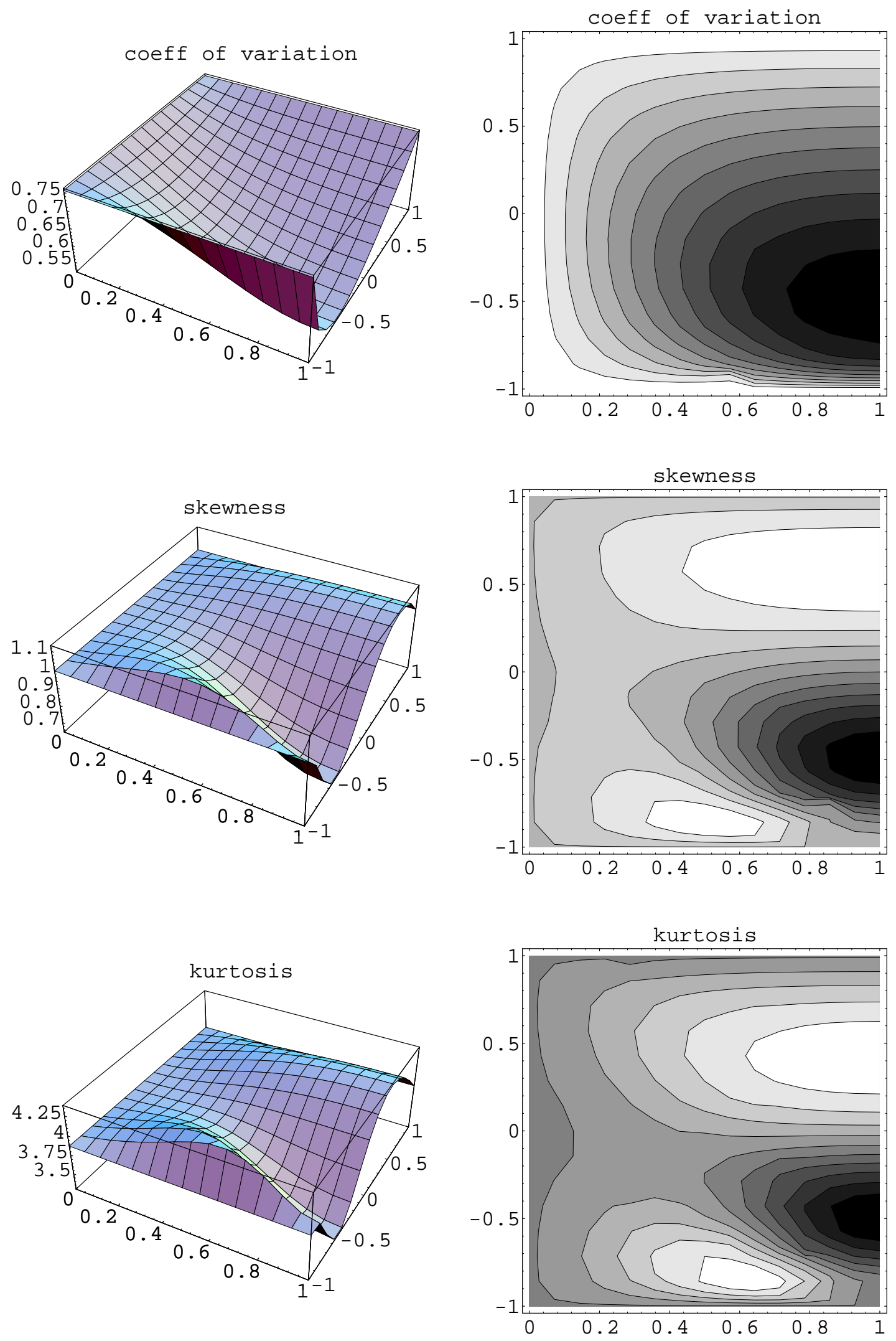

Figure 3: Statistics as functions of the traders' demands' variance ratio (from 0 to 1 ) and correlation (from -1 to 1 ). 

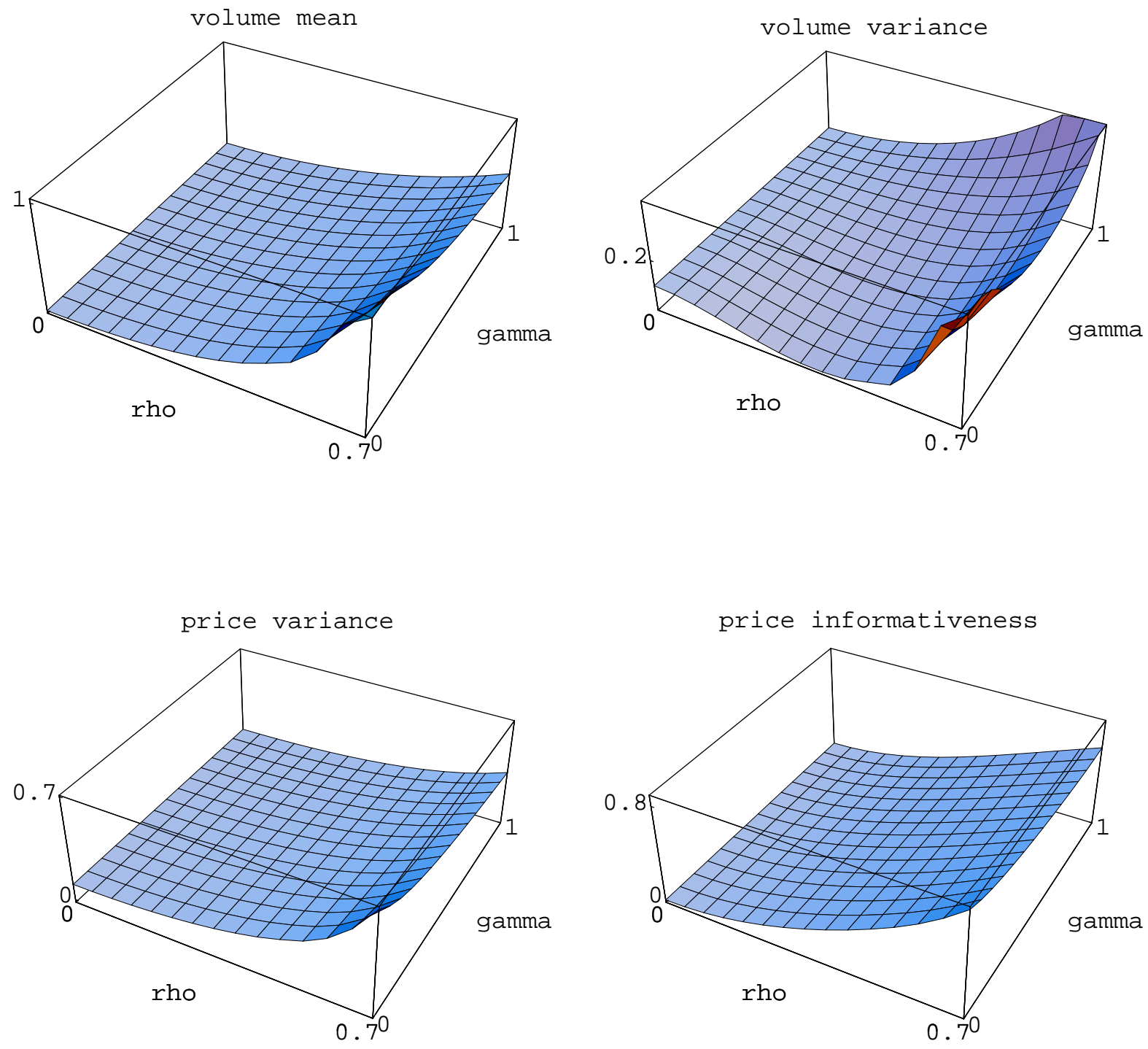

Figure 4: $\rho_{1}=\rho_{2}=\rho, \rho$ and $\gamma$ vary. 

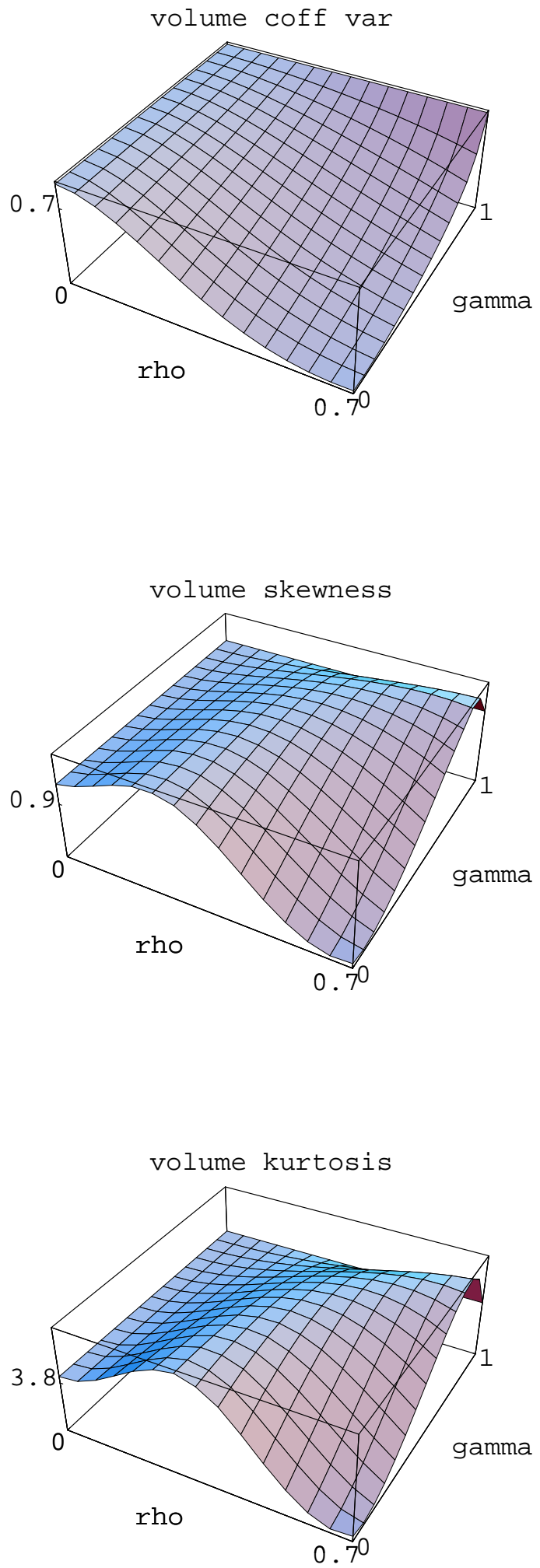

Figure 5: $\rho_{1}=\rho_{2}=\rho, \rho$ and $\gamma$ vary. 
volume mean

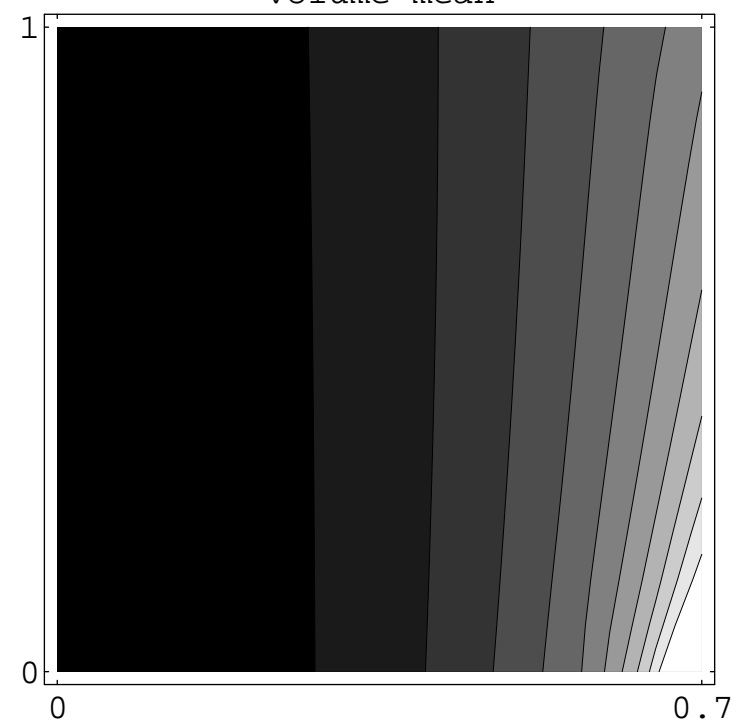

price variance

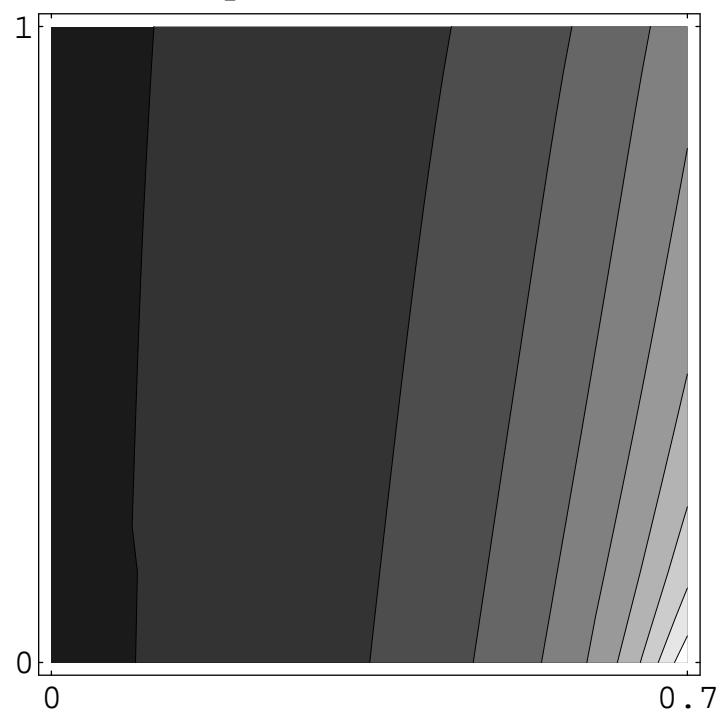

volume variance

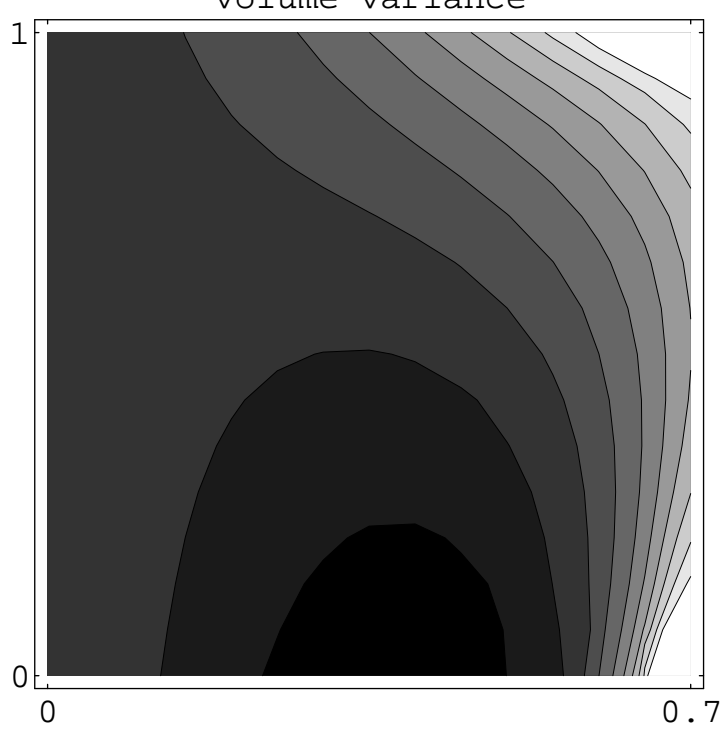

price informativeness

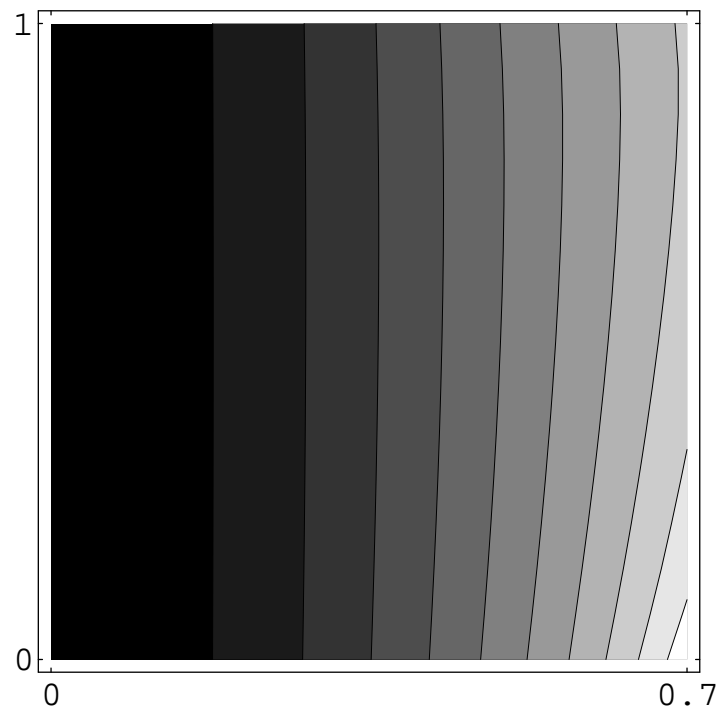

Figure 6: $\rho_{1}=\rho_{2}=\rho, \rho$ and $\gamma$ vary. 

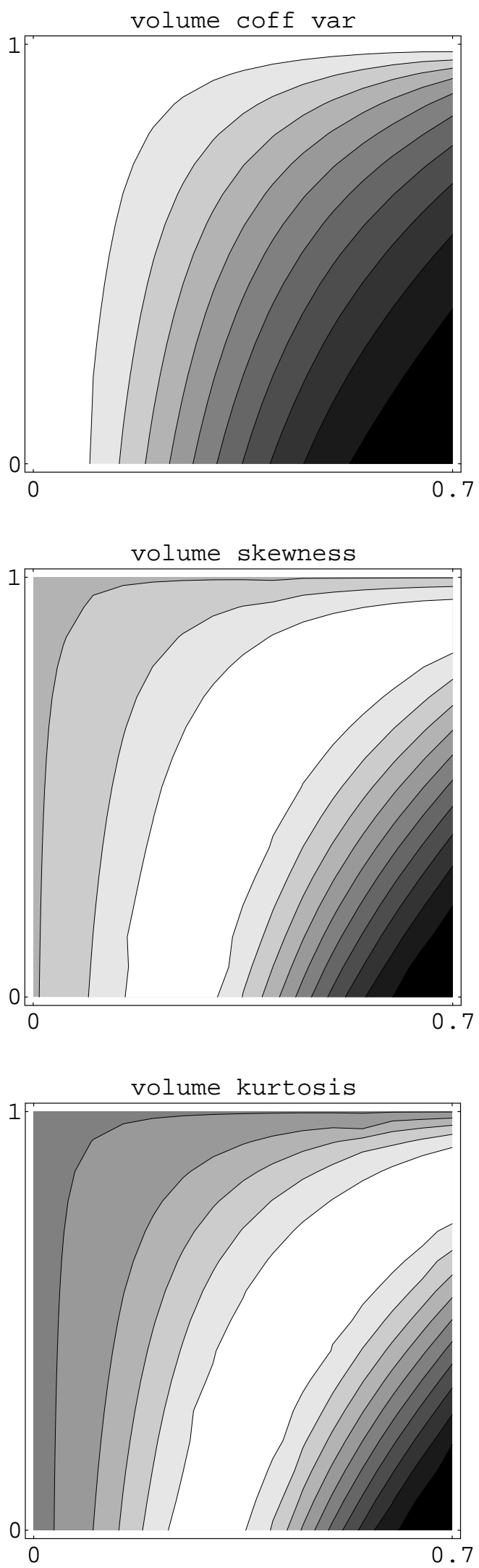

Figure 7: $\rho_{1}=\rho_{2}=\rho, \rho$ and $\gamma$ vary. 

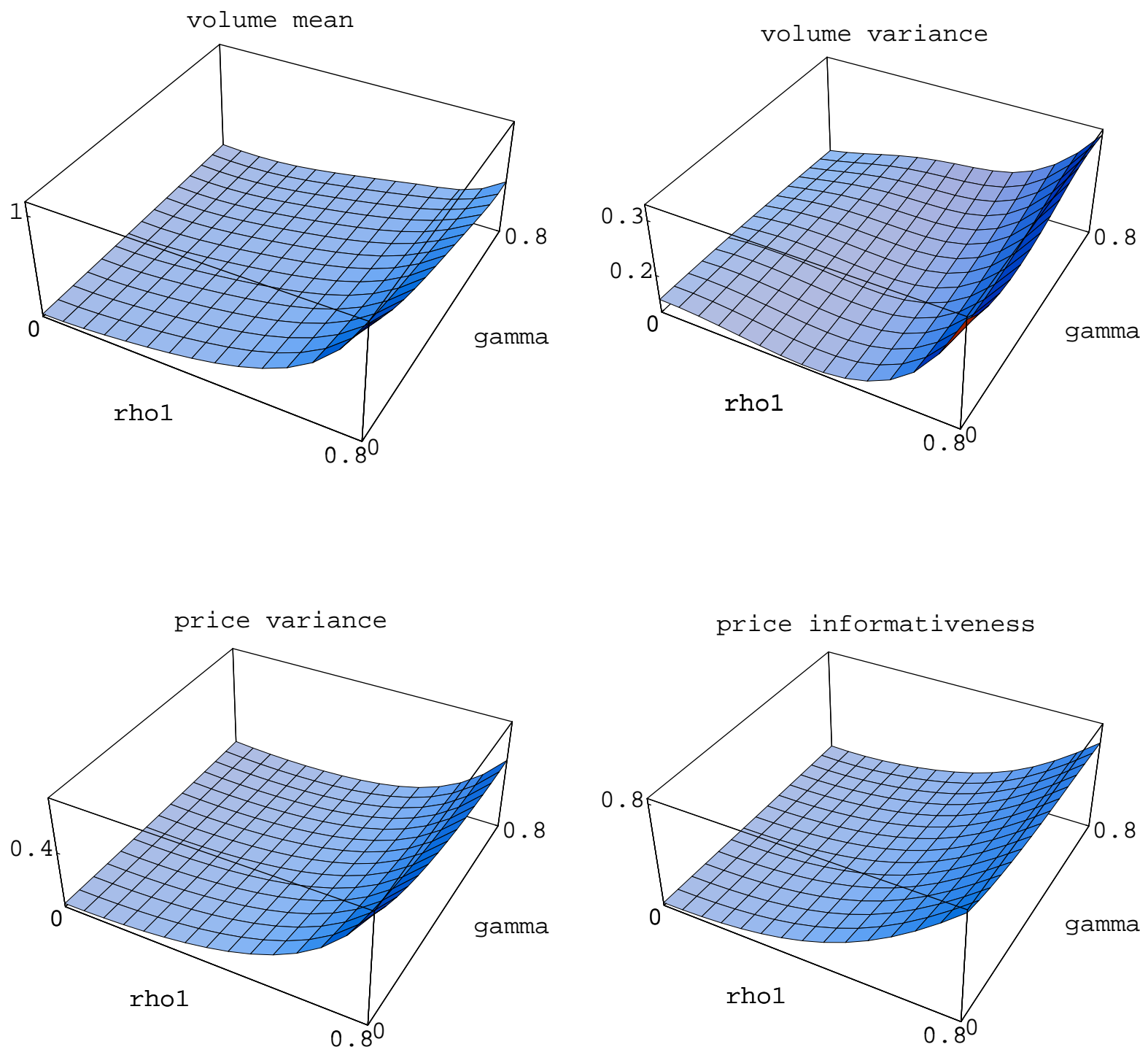

Figure 8: $\rho_{2}=.5, \rho 1$ and $\gamma$ vary. 

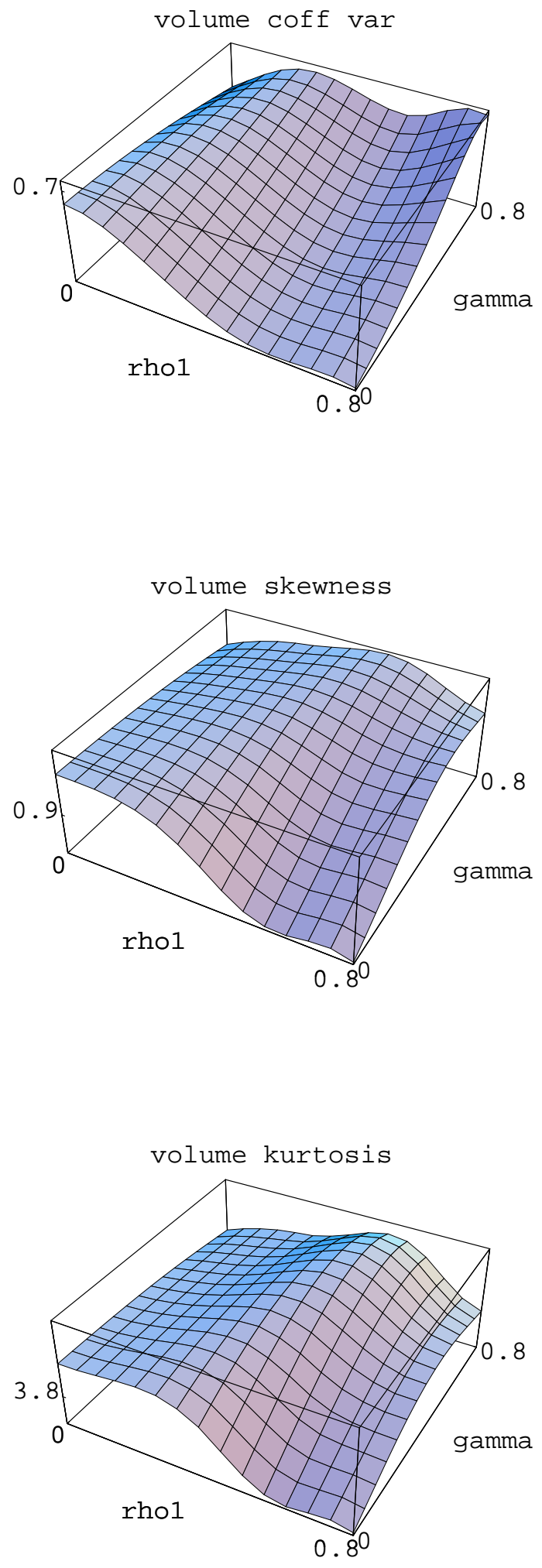

Figure 9: $\rho_{2}=.5, \rho 1$ and $\gamma$ vary. 
volume mean

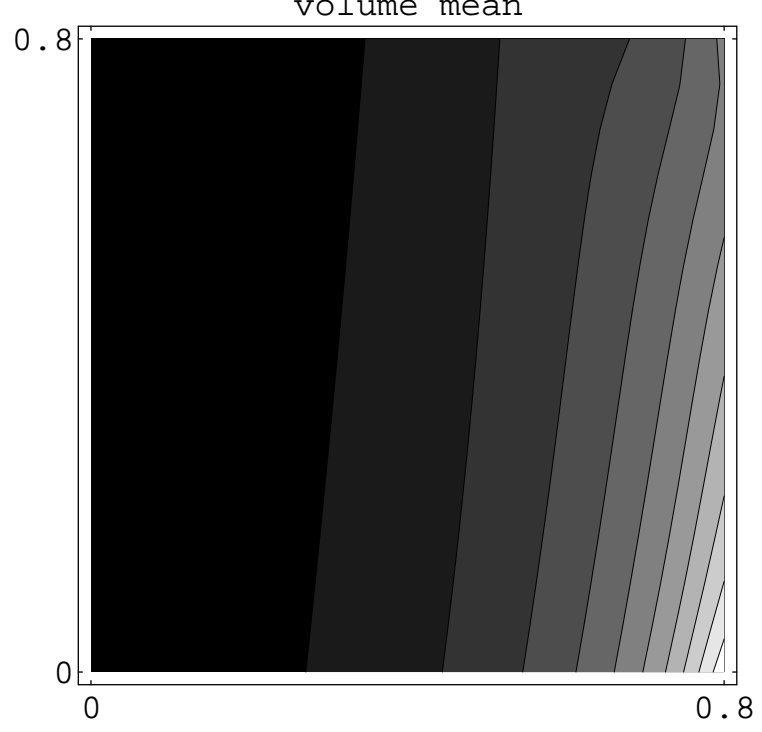

price variance

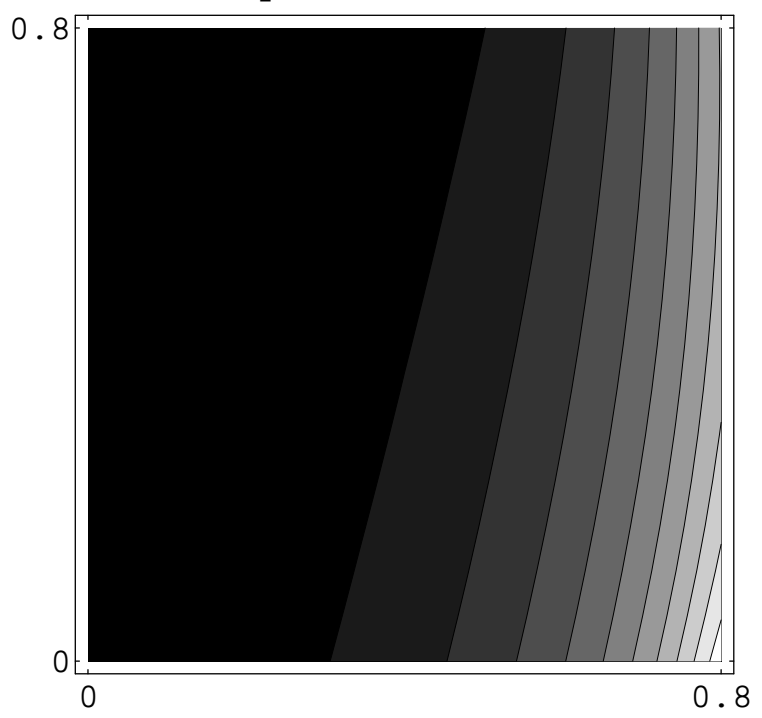

volume variance

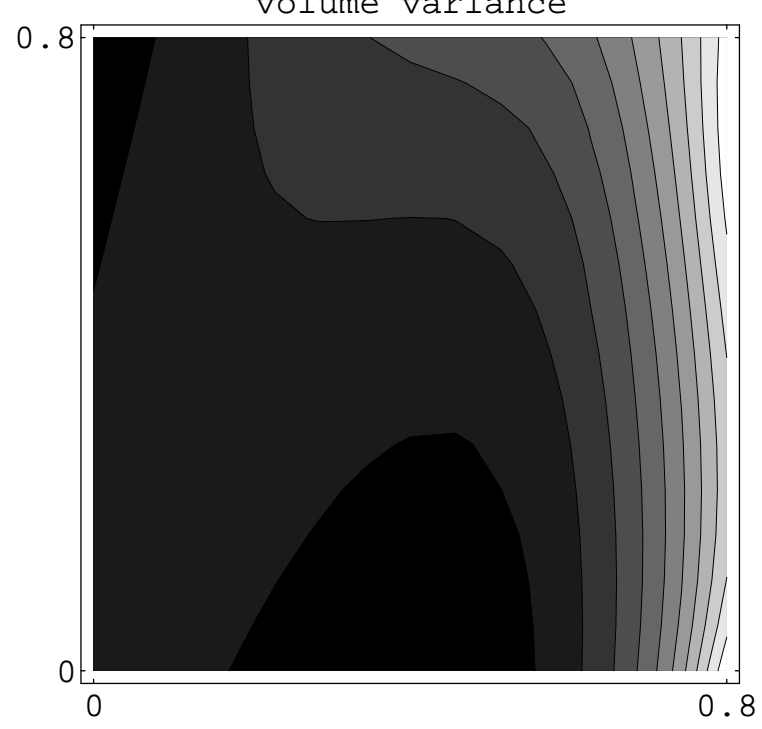

price informativeness

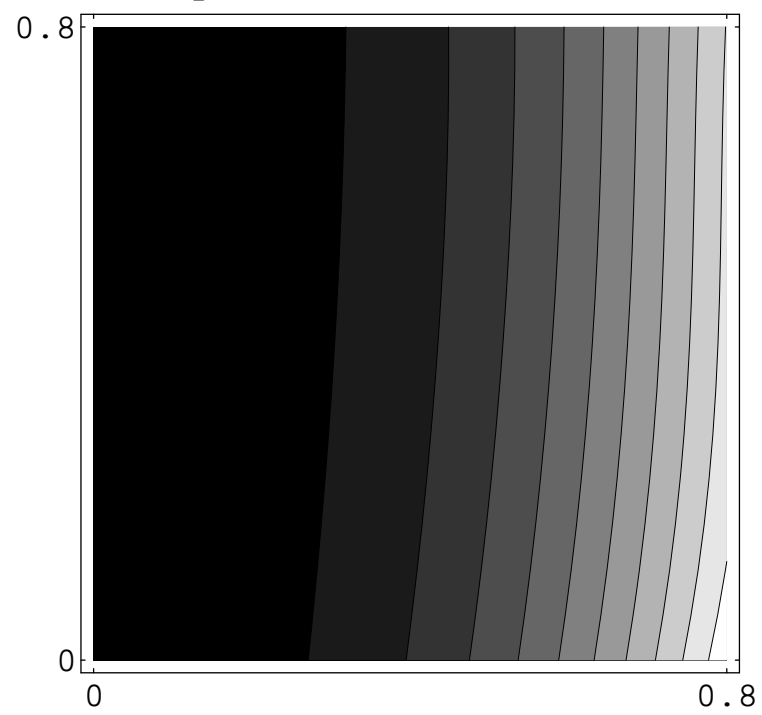

Figure 10: $\rho_{2}=.5, \rho 1$ and $\gamma$ vary. 

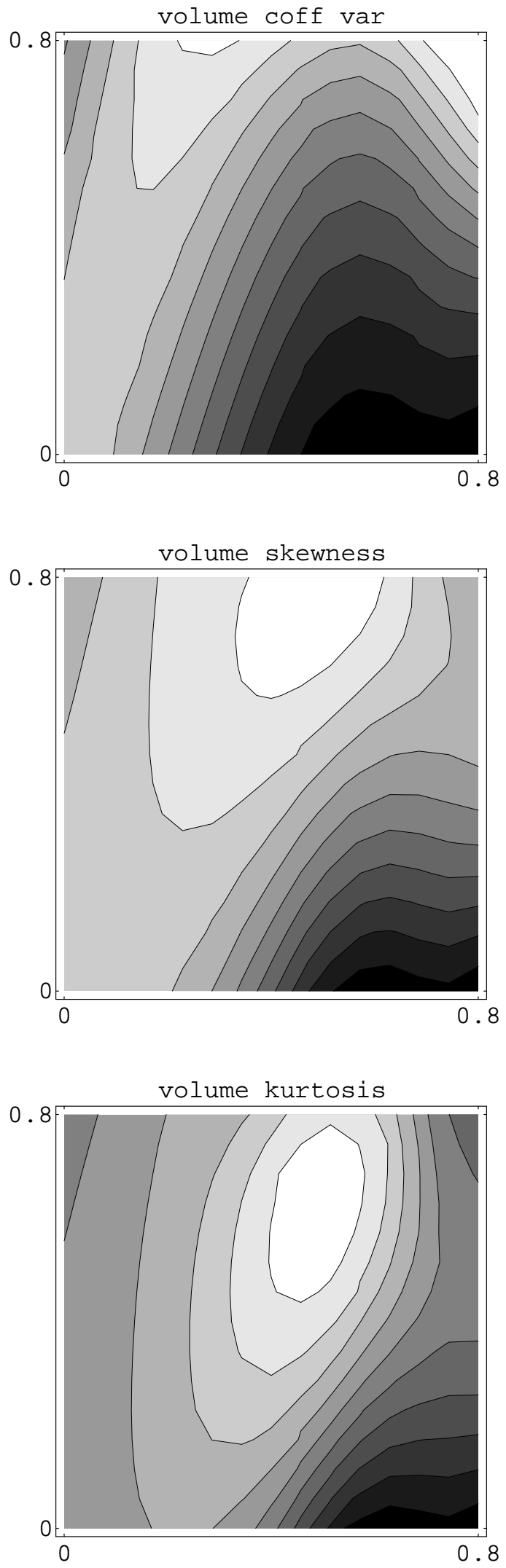

Figure 11: $\rho_{2}=.5, \rho 1$ and $\gamma$ vary. 

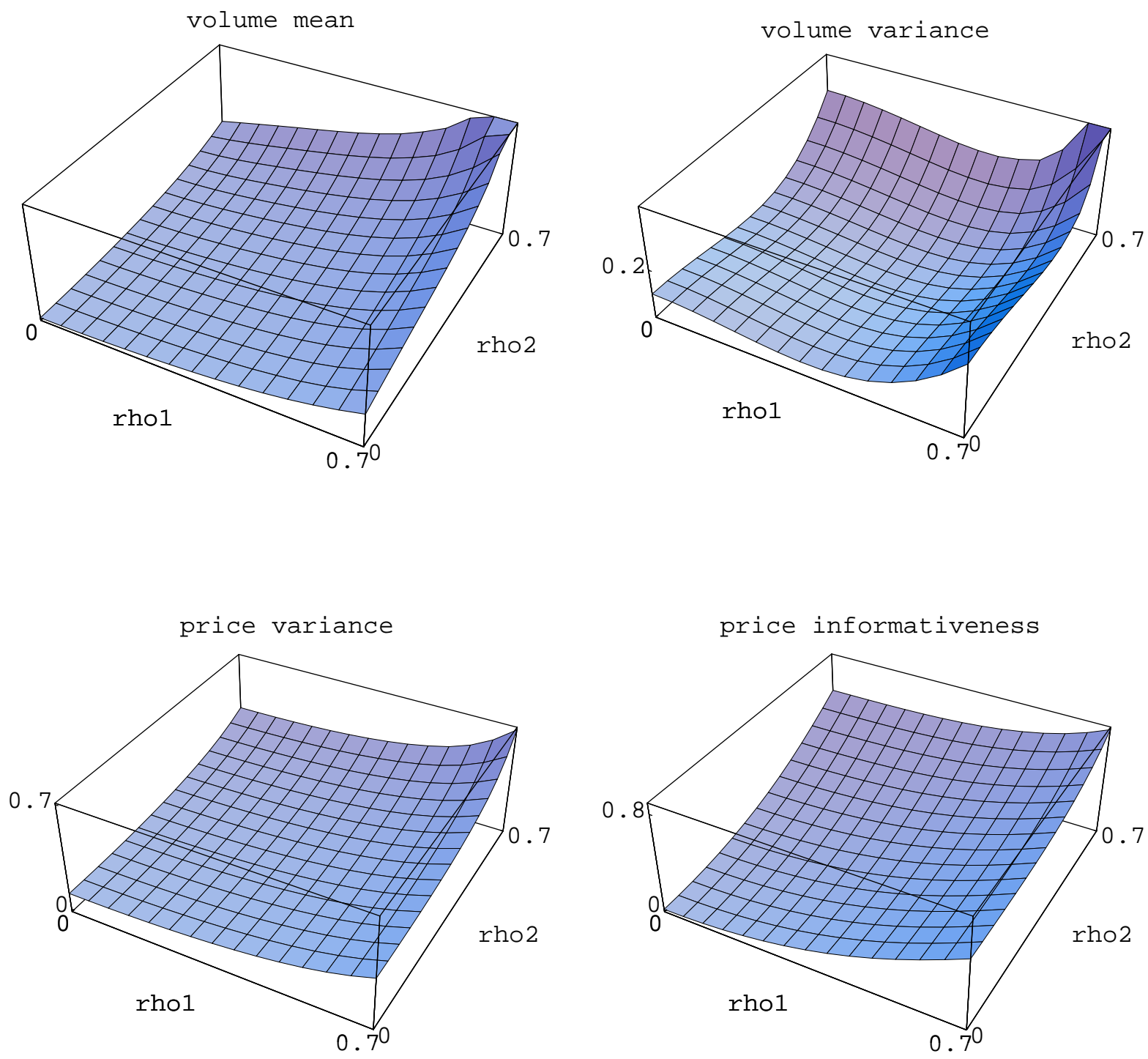

Figure 12: $\gamma=0, \rho_{1}$ and $\rho_{2}$ vary. 

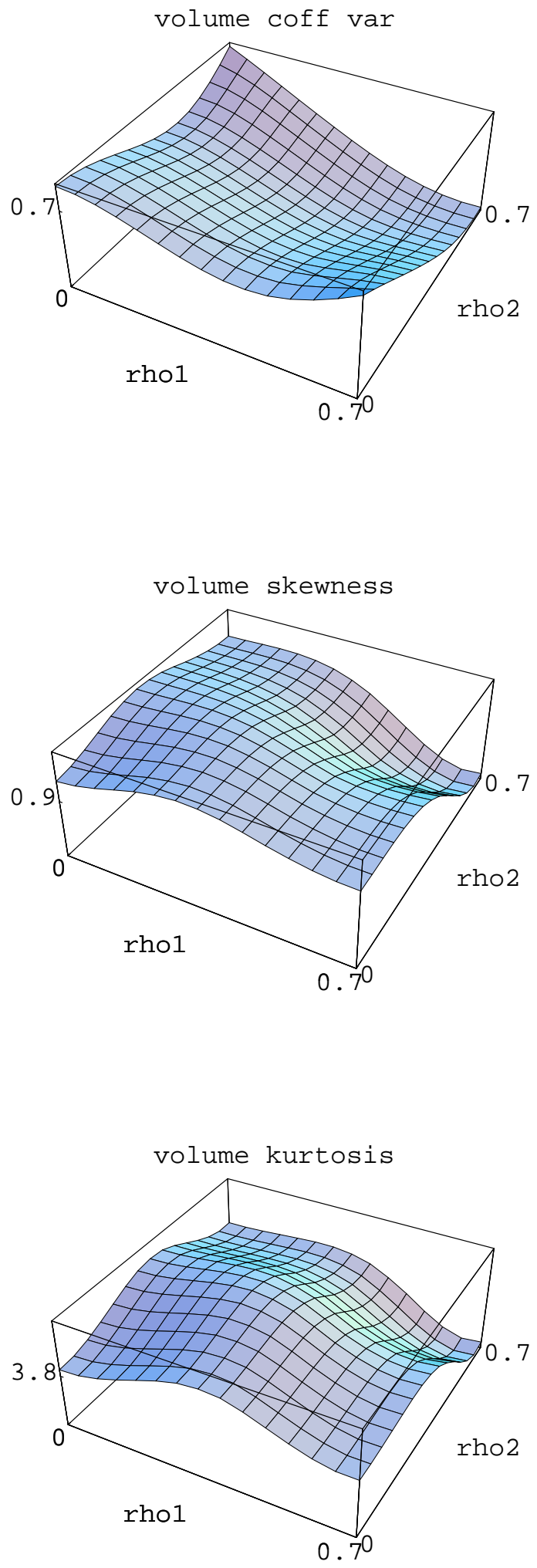

Figure 13: $\gamma=0, \rho_{1}$ and $\rho_{2}$ vary. 
volume mean

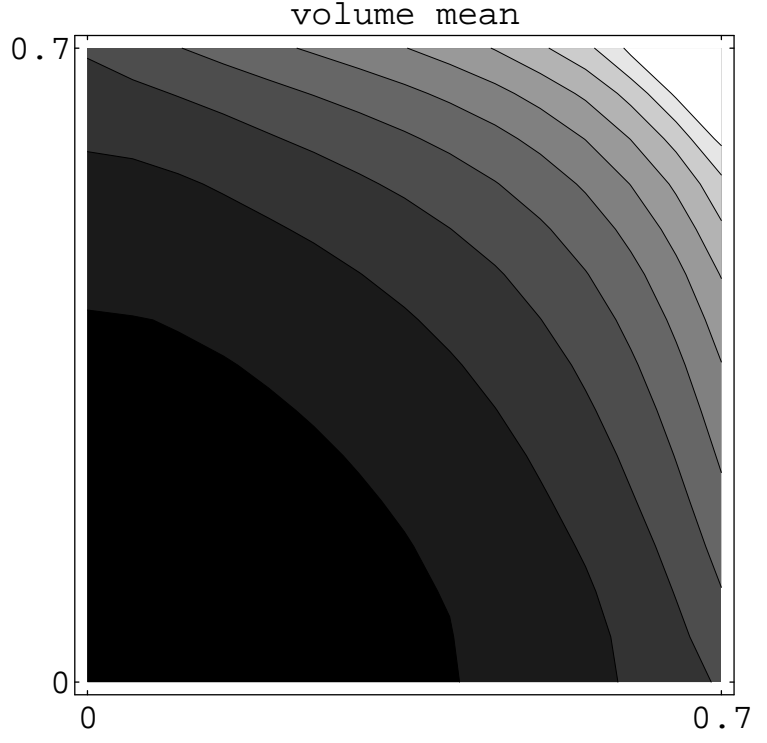

price variance

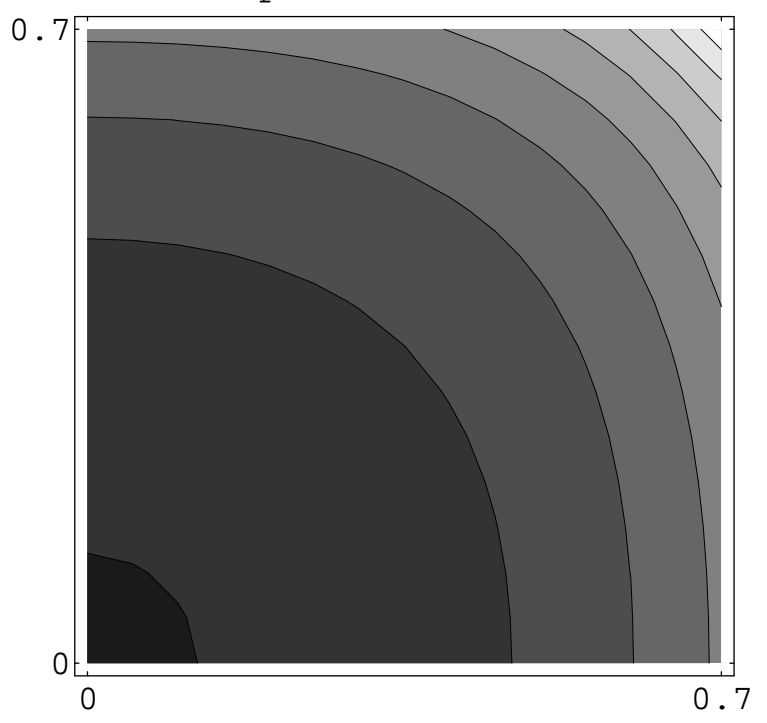

volume variance

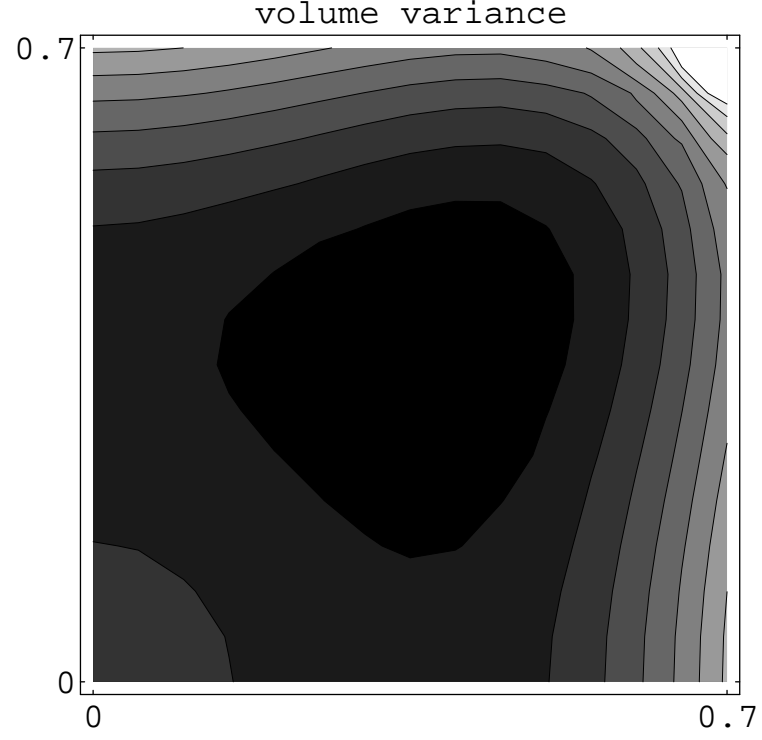

price informativeness

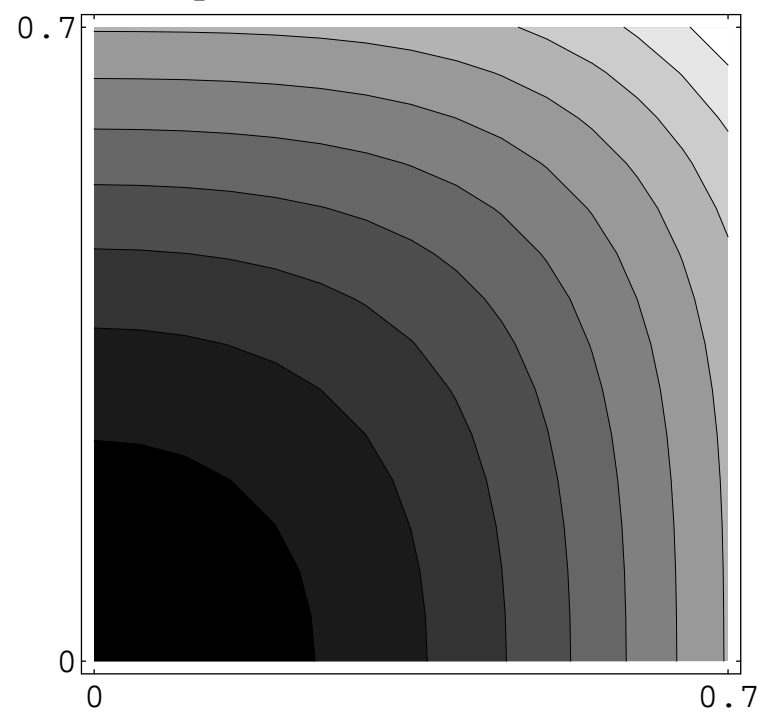

Figure 14: $\gamma=0, \rho_{1}$ and $\rho_{2}$ vary. 

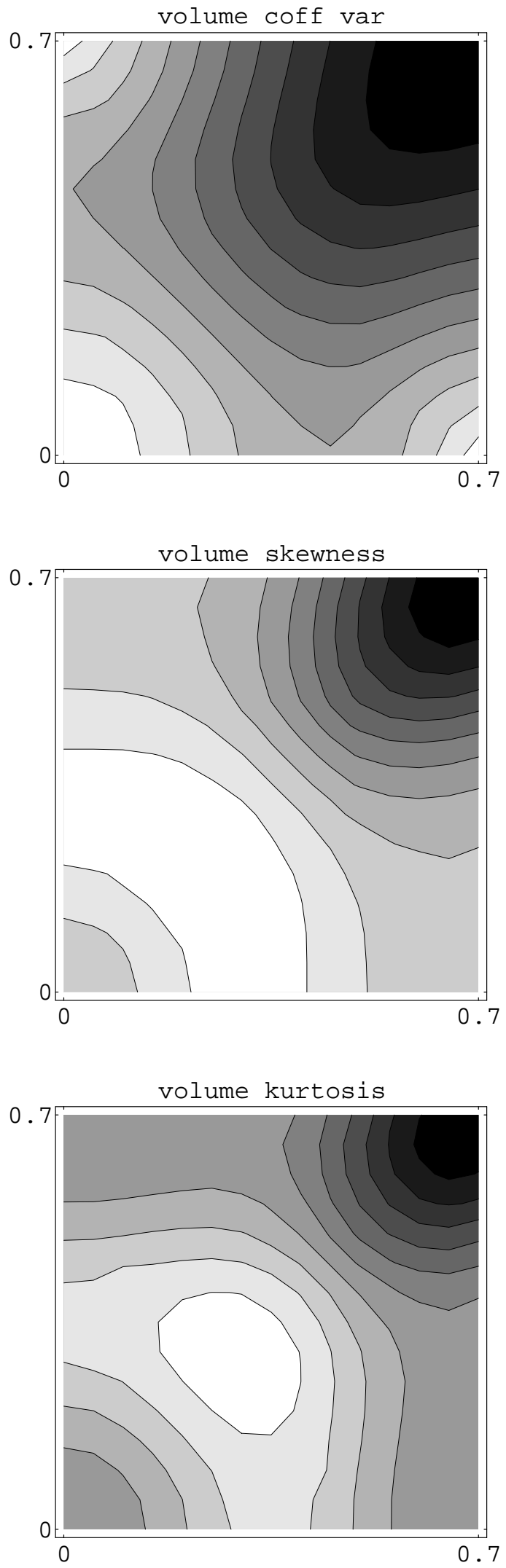

Figure 15: $\gamma=0, \rho_{1}$ and $\rho_{2}$ vary. 

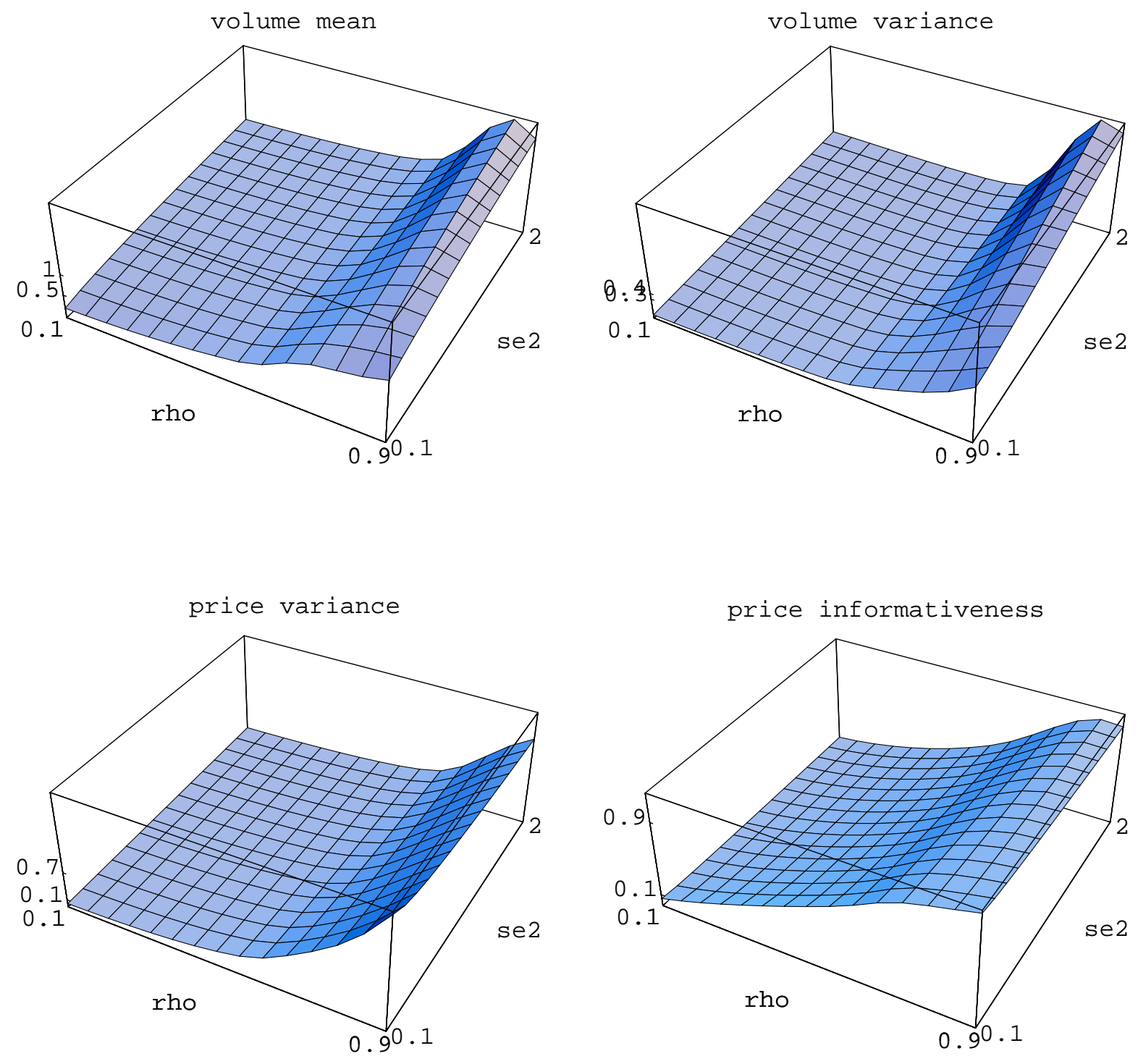

Figure 16: $\gamma=0, \rho_{1}=\rho_{2}=\rho, \rho$ and $\sigma_{\varepsilon}^{2}$ vary. 

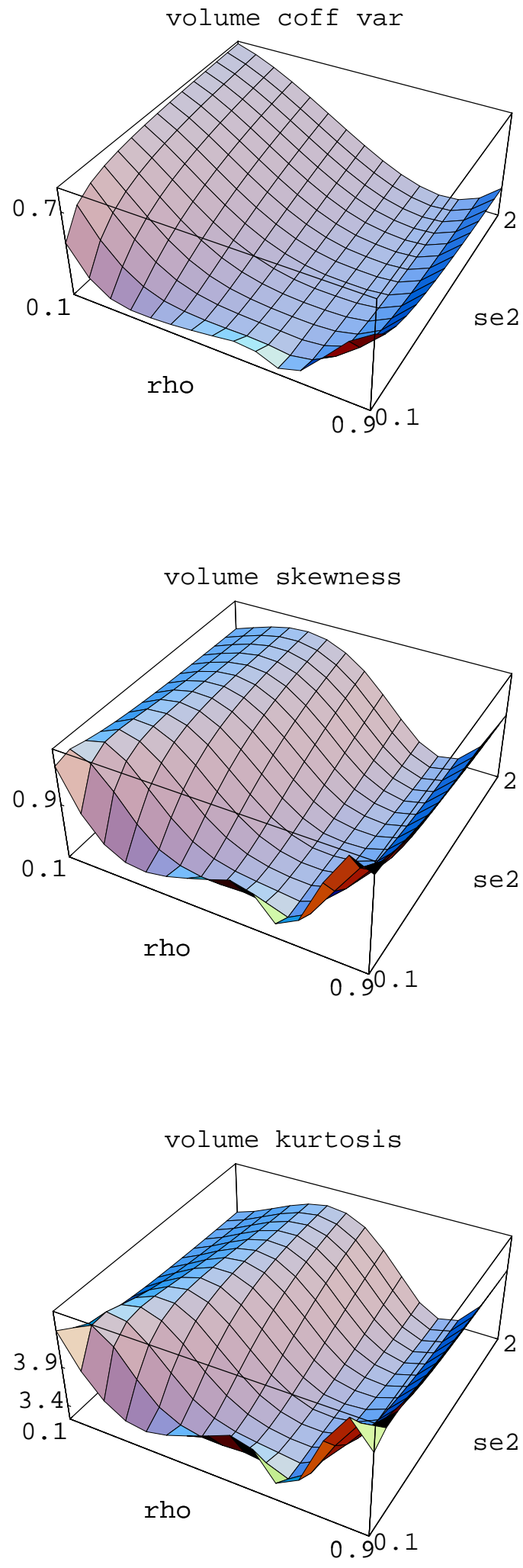

Figure 17: $\gamma=0, \rho_{1}=\rho_{2}=\rho, \rho$ and $\sigma_{\varepsilon}^{2}$ vary. 

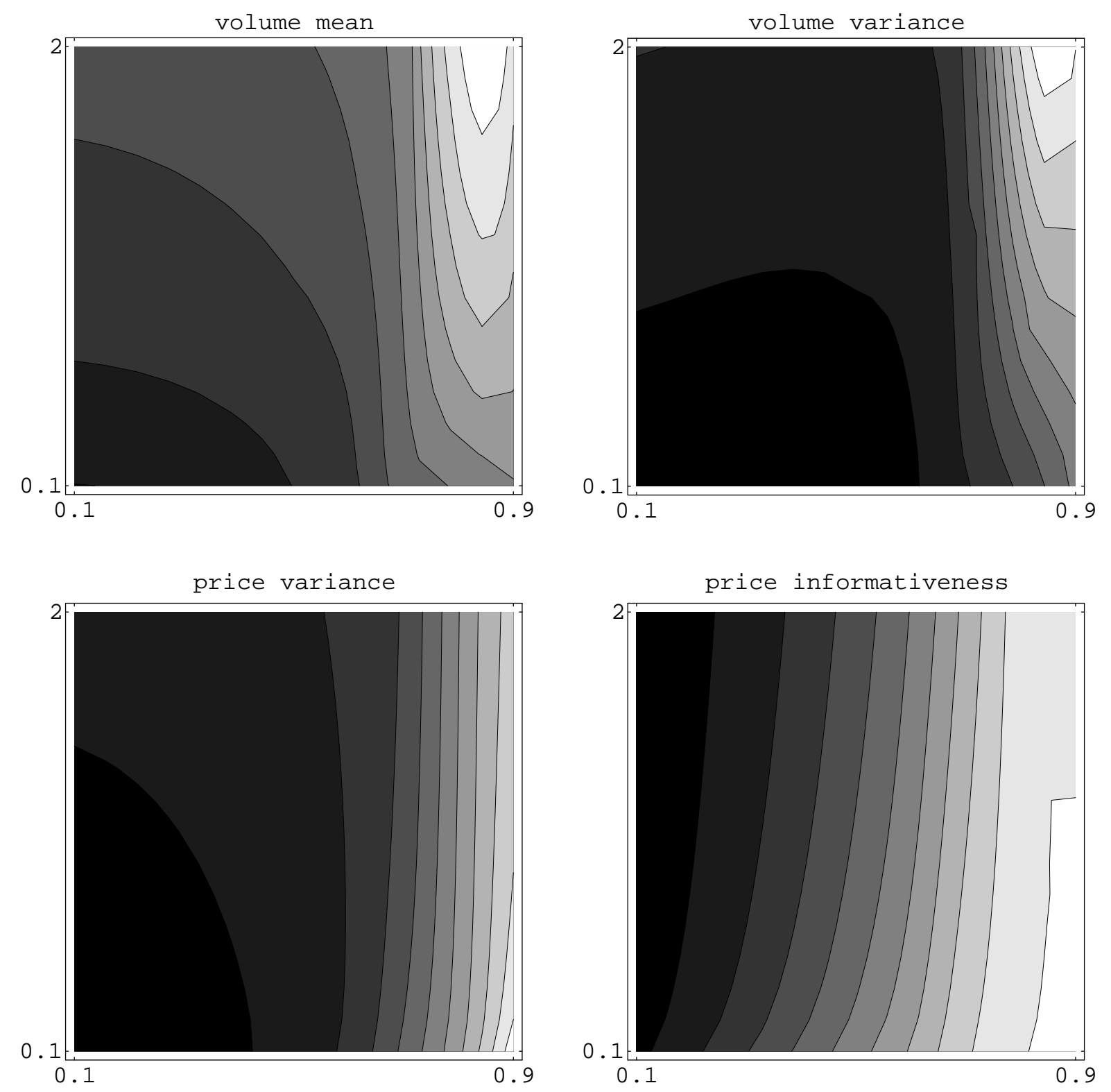

Figure 18: $\gamma=0, \rho_{1}=\rho_{2}=\rho, \rho$ and $\sigma_{\varepsilon}^{2}$ vary. 

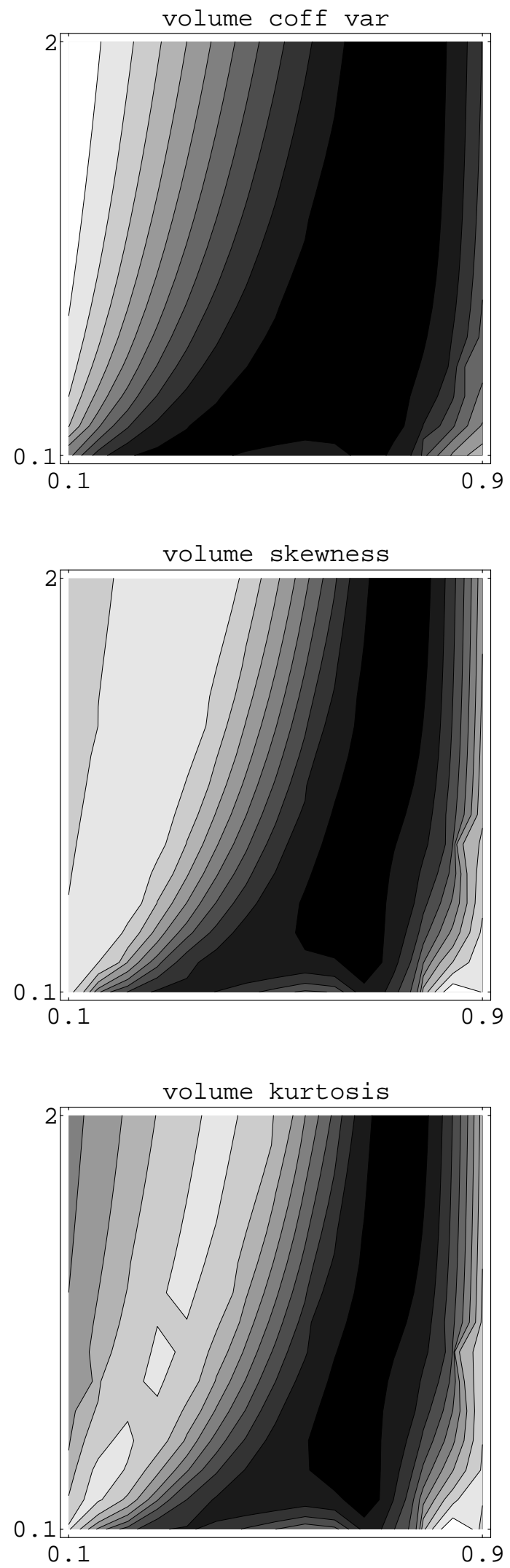

Figure 19: $\gamma=0, \rho_{1}=\rho_{2}=\rho, \rho$ and $\sigma_{\varepsilon}^{2}$ vary. 